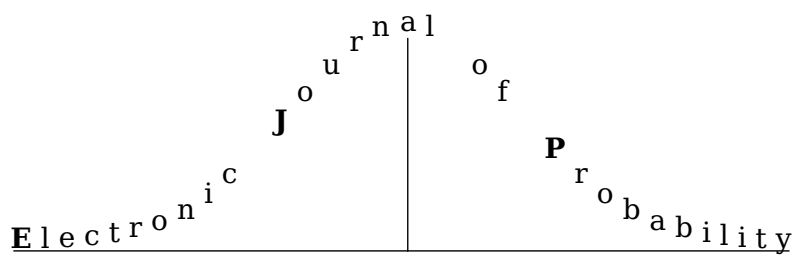

Electron. J. Probab. 25 (2020), article no. 93, 1-37.

ISSN: 1083-6489 https://doi.org/10.1214/20-EJP470

\title{
Smoothness and monotonicity of the excursion set density of planar Gaussian fields
}

\author{
Dmitry Beliaev* \\ Michael McAuley, ${ }^{*}$ \\ Stephen Muirhead ${ }^{\ddagger}$
}

\begin{abstract}
Nazarov and Sodin have shown that the number of connected components of the nodal set of a planar Gaussian field in a ball of radius $R$, normalised by area, converges to a constant as $R \rightarrow \infty$. This has been generalised to excursion/level sets at arbitrary levels, implying the existence of functionals $c_{E S}(\ell)$ and $c_{L S}(\ell)$ that encode the density of excursion/level set components at the level $\ell$. We prove that these functionals are continuously differentiable for a wide class of fields. This follows from a more general result, which derives differentiability of the functionals from the decay of the probability of 'four-arm events' for the field conditioned to have a saddle point at the origin. For some fields, including the important special cases of the Random Plane Wave and the Bargmann-Fock field, we also derive stochastic monotonicity of the conditioned field, which allows us to deduce regions on which $c_{E S}(\ell)$ and $c_{L S}(\ell)$ are monotone.
\end{abstract}

Keywords: Gaussian fields; nodal set; level sets; critical points. MSC2020 subject classifications: 60G60; 60G15; 58K05.

Submitted to EJP on July 15, 2019, final version accepted on May 26, 2020. Supersedes arXiv: 1905.09759.

\section{Introduction}

Let $f: \mathbb{R}^{2} \rightarrow \mathbb{R}$ be a continuous stationary Gaussian field with zero mean and covariance function $K: \mathbb{R}^{2} \rightarrow \mathbb{R}$ defined by $K(x)=\mathbb{E}(f(x) f(0))$. We are interested in the geometric properties of the (upper-)excursion sets and level sets of this field, defined respectively as

$$
\left\{x \in \mathbb{R}^{2}: f(x) \geq \ell\right\} \quad \text { and } \quad\left\{x \in \mathbb{R}^{2}: f(x)=\ell\right\}
$$

for $\ell \in \mathbb{R}$. Specifically, we are interested in the number of connected components of these sets in a large domain.

${ }^{*}$ Mathematical Institute, University of Oxford. E-mail: belyaev@maths . ox . ac.uk, mcauley@maths . ox .ac.uk

${ }^{\dagger}$ Present address: Department of Mathematics and Statistics, University of Helsinki.

E-mail: michael .mcauley@helsinki.fi

${ }^{\ddagger}$ School of Mathematical Sciences, Queen Mary University of London. E-mail: s. muirhead@qmul . ac. uk 
Smooth Gaussian fields are used to model spatial phenomena across wide ranging domains of science, such as quantum chaos, medical imaging and oceanography (see $[19,34,3]$ respectively). As a particular example; cosmological theories predict that the Cosmic Microwave Background Radiation observed on Earth can be well modelled as a realisation of a stationary Gaussian field on the two-dimensional sphere. Topological and geometric quantities provide a useful way of testing this prediction, which has important physical implications. In particular, [28] compares the number of excursion set components of the observed background radiation to the corresponding number for simulated Gaussian fields at a range of levels. A better understanding of the statistical properties of the number of excursion sets of Gaussian fields could therefore have consequences for such analysis.

Unlike certain other geometric functionals (e.g. the volume or Euler characteristic of excursion sets), the number of connected components is inherently difficult to study because it is non-local: the number of components in a domain cannot be counted by partitioning the domain and simply counting the number of components in each subdomain. Nazarov and Sodin [26] used an ergodic argument to study the asymptotics of this quantity. Specifically, if $f$ is an ergodic Gaussian field satisfying some regularity assumptions, $B(R)$ is the ball of radius $R>0$ centred at the origin, and $N_{L S}(R, 0)$ is the number of components of the nodal set $\left\{x \in \mathbb{R}^{2}: f(x)=0\right\}$ contained in $B(R)$, then there is a constant $c_{L S}(0) \geq 0$ such that

$$
\frac{N_{L S}(R, 0)}{\pi R^{2}} \rightarrow c_{L S}(0)
$$

as $R \rightarrow \infty$, where convergence occurs in $L^{1}$ and almost surely. Although this result was stated only for the nodal set, the arguments in [26] go through verbatim for excursion/level sets at arbitrary levels $\ell$; the respective limiting constants, denoted by $c_{E S}(\ell)$ and $c_{L S}(\ell)$, can be interpreted as the density of excursion/level set components per unit area.

In this paper we consider properties of $c_{E S}(\ell)$ and $c_{L S}(\ell)$ viewed as functions of the level. It was shown in [6] that $c_{E S}$ and $c_{L S}$ are absolutely continuous. Our main results (Theorems 2.10, 2.11 and Corollary 2.18) show that, for a wide class of fields, the continuous differentiability of $c_{E S}$ and $c_{L S}$ at $\ell$ is equivalent to the statement that, if the field is conditioned to have a saddle point at the origin at level $\ell$, then almost surely the 'arms' of the saddle (i.e. the four level lines that emanate from the saddle point) do not connect the origin to infinity. Since we can prove that the latter property holds for many fields, we deduce the continuous differentiability of the density functionals.

Recent work has established that, in many circumstances, the geometry of Gaussian excursion sets exhibits similar behaviour to that of discrete percolation models [4]. In particular, for a wide class of fields, it has been shown that the connectivity of the excursion sets exhibits a sharp phase transition at $\ell=0[30,25]$. Our results can therefore be compared to what is known, and conjectured, about the analogous density functionals for discrete percolation models. Consider Bernoulli bond percolation on the integer lattice, defined by declaring the edges of $\mathbb{Z}^{d}$ to be open independently with probability $p$ and closed otherwise (see [16] for background on this model). Let $K_{n}$ denote the number of open clusters that are contained in $[-n, n]^{d}$. Then it is known ([16, Chapter 4]) that

$$
\frac{K_{n}}{(2 n)^{d}} \rightarrow \kappa(p)
$$

as $n \rightarrow \infty$, almost surely and in $L^{1}$. This is a direct analogue of (1.1), and is also proven using an ergodic argument. The smoothness of $\kappa$ is of interest because it is related to the percolation phase transition. Specifically, it is conjectured in the physics literature 
that $\kappa$ is analytic on $[0,1] \backslash\left\{p_{c}\right\}$ and twice but not three times differentiable at $p_{c}$, where $p_{c} \in(0,1)$ is the critical probability for the model; this reflects the values of certain 'critical exponents' which are believed to be universal for percolation models (see [16, Chapter 9]). What has been shown rigorously, is that, for all $d \geq 2, \kappa$ is analytic on $\left[0, p_{c}\right)$ and smooth on $\left(p_{c}, 1\right]$, and in the case $d=2$ it is further known that $\kappa$ is analytic on $\left(p_{c}, 1\right]$ and at least twice differentiable at $p_{c}$ (see [16, Chapter 4]). Somewhat weaker results have been derived for other percolation models, including the Poisson-Boolean model and 'spread-out' percolation models [9].

Since the connectivity of the excursion sets of a wide class of planar Gaussian fields is conjectured, and in some cases known, to undergo a phase transition at $\ell=0$ that is analogous to the phase transition at $p_{c}$ for Bernoulli percolation (see $[4,7,29,30,25]$ ), it is natural to conjecture that, for such fields, $c_{E S}$ and $c_{L S}$ are also analytic on $\mathbb{R} \backslash\{0\}$ and twice but not three times differentiable at 0 . Our proof of the continuous differentiability of $c_{E S}$ and $c_{L S}$ can be seen as a first step in this direction.

Despite the connections to classical percolation theory, the method we use to prove differentiability of the density functionals is quite different. In Bernoulli percolation, the starting point is the equality

$$
\kappa(p)=\mathbb{E}_{p}\left(|C|^{-1}\right),
$$

where $|C|$ is the number of vertices in the open cluster at the origin. By enumerating clusters, this can be expressed as a power series in $p$, and the smoothness of $\kappa$ can be deduced from bounds on the coefficients in terms of connection probabilities for the cluster at the origin.

This approach does not readily generalise to the setting of Gaussian fields: whilst it can be shown that

$$
c_{E S}(\ell)=\mathbb{E}\left(\operatorname{Vol}(C)^{-1} \mathbb{1}_{f(0)>\ell}\right),
$$

where $\operatorname{Vol}(C)$ is the volume of the component of $\left\{x \in \mathbb{R}^{2}: f(x) \geq \ell\right\}$ containing the origin, it is not known whether the density of $(\operatorname{Vol}(C), f(0))$ is jointly continuous ([8] studies a kind of 'ergodic' density for $\operatorname{Vol}(C)$ at the zero level). Instead, our proof of differentiability uses an integral representation for $c_{E S}$ and $c_{L S}$ that was developed in [6] (see Theorem 2.6), although we still rely on the decay of certain 'connection probabilities' for the field $f$ conditioned to have a saddle point at the origin. These connections are the equivalent of 'four-arm events' in percolation, which play an important role in this theory (e.g., in the analysis of noise sensitivity [15]).

Our study of the integral representation for $c_{E S}$ and $c_{L S}$ also allows us to derive certain montonicity properties of these functionals (see Propositions 2.20-2.22); these results are of independent interest, and are a key input to proving lower bounds on the variance of the number of excursion/level sets of Gaussian fields (see Remark 2.24).

\section{Main results}

Throughout the paper we consider a planar Gaussian field satisfying the following assumption:

Assumption 2.1. The Gaussian field $f: \mathbb{R}^{2} \rightarrow \mathbb{R}$ is stationary and centred with $\operatorname{Var}(f(0))=1$ and satisfies;

1. The covariance function $K \in C^{4+\eta^{\prime}}$ for some $\eta^{\prime}>0$,

2. $\nabla^{2} f(0)$ is a non-degenerate Gaussian vector (it is conventional to treat $\nabla^{2} f(0)$ as a three-dimensional vector, ignoring degeneracy due to symmetry),

3. For any $x \in \mathbb{R}^{2}$, if $f(x)-f(0)$ is a non-degenerate Gaussian variable then the Gaussian vector $(\nabla f(x), \nabla f(0), f(x)-f(0))$ is non-degenerate. 
By Kolmogorov's theorem ([1, Theorem 1.4.2] and [17, Theorem 3.17]), $K \in C^{4+\eta^{\prime}}$ implies that $f \in C_{\text {loc }}^{2+\eta}\left(\mathbb{R}^{2}\right)$ almost surely for any $\eta \in\left(0, \eta^{\prime} / 2\right)$, and we fix such an $\eta$ for our analysis.

Since $K$ is continuous and positive definite, Bochner's theorem [10] states that it is the Fourier transform of a measure $\mu$ which is known as the spectral measure of the field:

$$
K(x)=\int_{\mathbb{R}^{2}} e^{i t \cdot x} d \mu(t)
$$

We can alternatively state our assumptions in terms of the spectral measure: $K \in C^{4+\eta^{\prime}}$ is equivalent to $\int_{\mathbb{R}^{2}}|t|^{4+\eta^{\prime}} d \mu(t)<\infty$. The second and third parts of Assumption 2.1 are equivalent to some non-degeneracy of the support of $\mu$ (see Appendix A).

We have in mind two important examples of Gaussian fields satisfying Assumption 2.1: (1) The Random Plane Wave (RPW), with covariance $K(x)=J_{0}(|x|)$, where $J_{0}$ is the 0 -th Bessel function, and spectral measure equal to the normalised Lebesgue measure on the unit circle; and (2) The Bargmann-Fock (BF) field, with covariance $K(x)=$ $\exp \left(-|x|^{2} / 2\right)$, and Gaussian spectral measure. The RPW is a universal model for high energy eigenfunctions of the Laplacian, see [11] for background. The BF field can be viewed as a continuous analogue of Bernoulli percolation, since it has rapid correlation decay and satisfies the FKG inequality, see [4] for details and further motivation.

We now formally define the density functionals $c_{E S}$ and $c_{L S}$. Let $N_{E S}(R, \ell)$ and $N_{L S}(R, \ell)$ denote respectively the number of components of $\left\{x \in \mathbb{R}^{2}: f(x) \geq \ell\right\}$ and $\left\{x \in \mathbb{R}^{2}: f(x)=\ell\right\}$ contained in $B(R)$ (i.e. the components which intersect $B(R)$ but not $\mathbb{R}^{2} \backslash B(R)$ ). Then the following asymptotic laws are known to hold:

Theorem 2.2 ([26, 22, 6]). Let $f$ be a Gaussian field satisfying Assumption 2.1. For each $\ell \in \mathbb{R}$, there exist $c_{E S}(\ell), c_{L S}(\ell) \geq 0$ such that

$$
\mathbb{E}\left[N_{E S}(R, \ell)\right]=c_{E S}(\ell) \cdot \pi R^{2}+O(R) \quad \text { and } \quad \mathbb{E}\left[N_{L S}(R, \ell)\right]=c_{L S}(\ell) \cdot \pi R^{2}+O(R)
$$

as $R \rightarrow \infty$. The constants implied by the $O(\cdot)$ notation are independent of $\ell$. If $f$ is also ergodic, then

$$
\frac{N_{E S}(R, \ell)}{\pi R^{2}} \rightarrow c_{E S}(\ell) \text { and } \frac{N_{L S}(R, \ell)}{\pi R^{2}} \rightarrow c_{L S}(\ell)
$$

as $R \rightarrow \infty$, almost surely and in $L^{1}$.

Remark 2.3. The notation in [22] and elsewhere is slightly different: $c_{L S}$ in the present paper is denoted $c_{N S}$ in some previous papers.

In [6] a representation of $c_{E S}$ and $c_{L S}$ was given in terms of the densities of certain types of critical points. To state this we introduce upper/lower connected saddle points.

Definition 2.4. Let $x_{0}$ be a saddle point of a $C^{2}$ function $g: \mathbb{R}^{2} \rightarrow \mathbb{R}$ such that there are no other critical points at the same level as $x_{0}$ (that is, if $x_{1}$ is another critical point of $g$, then $g\left(x_{1}\right) \neq g\left(x_{0}\right)$ ). We say that $x_{0}$ is upper connected if it is the closure of only one component of $\left\{x \in \mathbb{R}^{2}: g(x)>g\left(x_{0}\right)\right\}$. We say that $x_{0}$ is lower connected if it in the closure of only one component of $\left\{x \in \mathbb{R}^{2}: g(x)<g\left(x_{0}\right)\right\}$.

Interestingly, this definition was used as far back as 1870, by Maxwell [23], to understand topographical properties of landscapes.

It was shown in $[13,12]$ that the expected number of local maxima, local minima or saddle points of a Gaussian field with height in a certain range can be expressed as the integral of an explicit continuous density function over the height range. In [6] this result was extended to upper and lower connected saddle points without explicitly computing the corresponding density functions: 
Proposition 2.5 ([6, Proposition 1.8]). Let $f$ be a Gaussian field satisfying Assumption 2.1. Then there exist non-negative functions $p_{m^{+}}, p_{m^{-}}, p_{s^{+}}, p_{s^{-}}, p_{s} \in L^{1}(\mathbb{R})$ such that the following holds. Let $\Omega \subset \mathbb{R}^{2}$ be compact and $\partial \Omega$ have finite Hausdorff- 1 measure. Let $\ell \in \mathbb{R}$ and let $N_{m^{+}}(\ell), N_{m^{-}}(\ell), N_{s^{+}}(\ell), N_{s^{-}}(\ell)$ and $N_{s}(\ell)$ denote the number of local maxima, local minima, upper connected saddles, lower connected saddles and saddles of $f$ in $\Omega$ with level above $\ell$ respectively. Then

$$
\mathbb{E}\left[N_{h}(\ell)\right]=\operatorname{Area}(\Omega) \int_{\ell}^{\infty} p_{h}(x) d x
$$

for $h=m^{+}, m^{-}, s^{+}, s^{-}, s$. Furthermore, these functions can be chosen to satisfy the relations $p_{m^{+}}(x)=p_{m^{-}}(-x), p_{s^{+}}(x)=p_{s^{-}}(-x)$ and $p_{s^{-}}+p_{s^{+}}=p_{s}$, and such that $p_{m^{+}}$, $p_{m^{-}}$and $p_{s}$ are continuous.

We can now state the main result of [6], characterising $c_{E S}$ and $c_{L S}$ in terms of the densities in Proposition 2.5:

Theorem 2.6 ([6, Theorem 1.9]). Let $f$ be a Gaussian field satisfying Assumption 2.1, and let $p_{m^{+}}, p_{m^{-}}, p_{s^{+}}, p_{s^{-}}$denote the densities specified in Proposition 2.5. Then

$$
c_{E S}(\ell)=\int_{\ell}^{\infty} p_{m^{+}}(x)-p_{s^{-}}(x) d x
$$

and

$$
c_{L S}(\ell)=\int_{\ell}^{\infty} p_{m^{+}}(x)-p_{s^{-}}(x)+p_{s^{+}}(x)-p_{m^{-}}(x) d x,
$$

and hence $c_{E S}$ and $c_{L S}$ are absolutely continuous.

One of the motivations for Theorem 2.6 was to provide a tool with which to study the excursion/level set densities: since $p_{m^{+}}, p_{m^{-}}$, and $p_{s}=p_{s^{+}}+p_{s^{-}}$are explicitly known for a wide class of fields, by establishing simple properties of $p_{s^{-}}$we can deduce results for $c_{E S}$ and $c_{L S}$. We expand upon this method in this paper. Specifically, we consider the function

$$
p_{s^{-}}^{*}(\ell):=p_{s}(\ell) \mathbb{P}\left(\tilde{f}_{\ell} \text { has a lower connected saddle point at the origin }\right),
$$

where $\tilde{f}_{\ell}$ is the field $f$ conditioned to have a saddle point at the origin at level $\ell$ (in the sense of Palm distributions; see Lemma 3.2 for a formal definition). Under mild conditions we show that $p_{s^{-}}^{*}$ defines a version of $p_{s^{-}}$(recall that the latter is defined only up to null sets). By studying $\tilde{f}_{\ell}$ we are able to deduce properties of $p_{s^{-}}^{*}$, and hence of $c_{E S}$ and $c_{L S}$.

\subsection{Differentiability}

Our first set of results concerns the differentiability of $c_{E S}$ and $c_{L S}$. Let us begin by detailing the necessary assumptions on $f$.

Assumption 2.7. For all $t \in \mathbb{R}^{2} \backslash\{0\}$,

$$
\operatorname{Cov}\left((f(t), \nabla f(t)) \mid f(0), \nabla f(0), \nabla^{2} f(0)\right)
$$

is non-degenerate (i.e. this $3 \times 3$ matrix has non-zero determinant).

Assumption 2.8. There exist $c, \nu>0$ such that, for all $|t| \geq 1$,

$$
\max _{|k| \leq 3}\left|\partial^{k} K(t)\right| \leq c|t|^{-(1+\nu)} .
$$

Moreover, there exists a neighbourhood $V$ of the origin on which the spectral measure $\mu$ has density $\rho$ with respect to the Lebesgue measure and $\inf _{V} \rho>0$. 
Assumption 2.9. For $0<r<R$, let $\operatorname{Arm}_{\ell}(r, R)$ denote the 'one-arm event' that there exists a component of $\{f \geq \ell\}$ which intersects both $\partial B(r)$ and $\partial B(R)$. Then there exist $c_{1}, c_{2}>0$ such that for any $1<r<R$

$$
\mathbb{P}\left(f \in \operatorname{Arm}_{0}(r, R)\right) \leq c_{1}(r / R)^{c_{2}} .
$$

Assumption 2.7 is extremely mild; it is satisfied whenever the support of the spectral measure $\mu$ is not too degenerate. It is sufficient for this support to contain an open set or an ellipse/circle (Lemma A.2), so in particular, it holds for the RPW and the BF field.

Assumptions 2.8 and 2.9 are somewhat more restrictive. Assumption 2.8 holds for any smooth field with sufficiently nice correlation decay, and in particular holds for the BF field, but it does not hold for the RPW (whose correlations decay only as $|t|^{-1 / 2}$ ). It also implies Assumption 2.7, by the previous remark.

Assumption 2.9 relates to the conjectured properties of the 'percolation universality class', and has been shown to hold for a wide class of fields that includes the BF field $[4,30]$. Moreover it is strongly believed to hold for the RPW. We state our results directly in terms of one-arm decay as it is likely that these bounds will be extended to more fields over time.

Our first main result is that $c_{E S}$ and $c_{L S}$ are continuously differentiable under the above assumptions:

Theorem 2.10. Suppose $f$ is a Gaussian field satisfying Assumptions 2.1 and 2.8-2.9 (e.g. the Bargmann-Fock field). Then $c_{E S}$ and $c_{L S}$ are continuously differentiable on $\mathbb{R}$. In other words, the functions $p_{s^{-}}$and $p_{s^{+}}$defined in Proposition 2.5 can be chosen to be continuous, and

$$
c_{E S}^{\prime}(\ell)=-p_{m^{+}}(\ell)+p_{s^{-}}(\ell)
$$

and

$$
c_{L S}^{\prime}(\ell)=-p_{m^{+}}(\ell)+p_{s^{-}}(\ell)-p_{s^{+}}(\ell)+p_{m^{-}}(\ell) .
$$

We emphasise that Theorem 2.10 applies to a wide class of fields, including the important case of the BF field, but does not apply to the RPW as stated (although we believe the conclusion to be true).

\subsubsection{Four-arm saddle points}

Theorem 2.10 follows from a more general result establishing that, under very mild conditions, the continuous differentiability of $c_{E S}$ and $c_{L S}$ is implied by the decay of certain connection probabilities involving 'four-arm saddles'.

Let $D \subset \mathbb{R}^{2}$ be a simply connected domain with piecewise $C^{1}$ boundary and let $x_{0} \in D$ be a saddle point of $g \in C^{2}\left(\mathbb{R}^{2}\right)$ such that $g$ has no other critical points at the same level as $x_{0}$. We say that $x_{0}$ is four-arm in $D$ if it is in the closure of two components of $\left\{x \in D: g(x)>g\left(x_{0}\right)\right\}$ and two components of $\left\{x \in D: g(x)<g\left(x_{0}\right)\right\}$ (see Figure 1(a)); intuitively, a saddle point is four-arm in $D$ if we cannot tell whether it is upper or lower connected by looking at the values of $g$ in $D$. A saddle point $x_{0}$ is said to be infinite four-arm if it is in the closure of two components of $\left\{x \in \mathbb{R}^{2}: g(x)>g\left(x_{0}\right)\right\}$ and two components of $\left\{x \in \mathbb{R}^{2}: g(x)<g\left(x_{0}\right)\right\}$ (see Figure 1(b)). As mentioned in Section 1, four-arm saddle points are analogous to four-arm events for percolation models.

Recall the conditional field $\tilde{f}_{\ell}$ (to be formally defined in Lemma 3.2 ) and the functions $p_{s^{-}}^{*}$ and $p_{s^{-}}$defined in (2.3) and Proposition 2.5 respectively. 


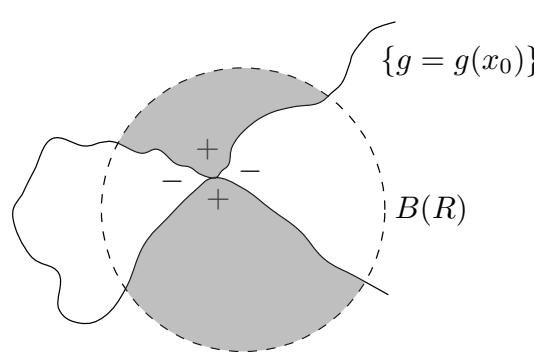

(a) An upper connected saddle point that is four-arm in $B(R)$.

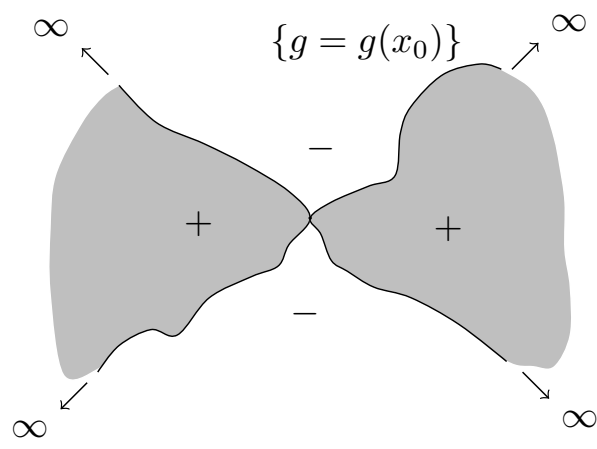

(b) An infinite-four-arm saddle point.

Figure 1

Theorem 2.11. Let $f$ be a Gaussian field satisfying Assumptions 2.1 and 2.7. Then $p_{s^{-}}^{*}=p_{s^{-}}$almost everywhere. Moreover, let $a<b$ and suppose that for all $\ell \in(a, b)$

$$
\mathbb{P}\left(\tilde{f}_{\ell} \text { has an infinite four-arm saddle at the origin }\right)=0 .
$$

Then $\left.p_{s^{-}}^{*}\right|_{(a, b)}$ is continuous, and so $c_{E S}$ and $c_{L S}$ are continuously differentiable on $(a, b)$.

Theorem 2.10 follows from Theorem 2.11 once we verify condition (2.5) under Assumptions 2.8 and 2.9. To do so, we use Assumption 2.8 and a Cameron-Martin argument to treat the conditional field $\tilde{f}_{\ell}$ away from the origin as a perturbation of the unconditioned field $f$. We then use Assumption 2.9 to bound the relevant connection probabilities for the unconditioned field.

As a corollary of Theorem 2.11 (actually of its proof), we deduce a bound on the number of saddle points of a Gaussian field that are four-arm inside a ball and whose level lies in a narrow range. This improves a bound that was previously established in [6], and is also a key ingredient in proving lower bounds on the variance of the number of excursion/level set components (see Remark 2.24).

Corollary 2.12. Let $f$ be a Gaussian field satisfying all the assumptions of Theorem 2.11. Then there exists a function $\delta_{R} \rightarrow 0$ as $R \rightarrow \infty$ and a constant $c>0$ such that, for each $R>1$ and $a \leq a_{R} \leq b_{R} \leq b$,

$$
\mathbb{E}\left(N_{4-\operatorname{arm}}\left(R,\left[a_{R}, b_{R}\right]\right)\right) \leq c \min \left\{\delta_{R} R^{2}\left(b_{R}-a_{R}\right), R\right\}
$$

where $N_{4 \text {-arm }}\left(R,\left[a_{R}, b_{R}\right]\right)$ is the number of saddle points of $f$ which are four-arm in $B(R)$ and have level in $\left[a_{R}, b_{R}\right]$.

Remark 2.13. In [6] it was shown that $\mathbb{E}\left(N_{4-\operatorname{arm}}(R)\right)=O(R)$; Corollary 2.12 supersedes this bound whenever $b_{R}-a_{R}=O\left(R^{-1}\right)$. It is possible to improve the conclusion of Corollary 2.12 further by imposing stronger assumptions on the field. For example, suppose we assume the exponential decay of arm probabilities at non-zero levels: for some $\ell^{*}>0$ and $\delta \in(0,1)$, there exist $c_{1}, c_{2}>0$ such that

$$
\mathbb{P}\left(f \in \operatorname{Arm}_{\ell^{*}}(\delta R, R)\right) \leq c_{1} e^{-c_{2} R} .
$$

Then for any $a>\ell^{*}$ (or $b<-\ell^{*}$ ), it is possible to prove that there exists $c>0$ such that

$$
\mathbb{E}\left(N_{4-\operatorname{arm}}\left(R,\left[a_{R}, b_{R}\right]\right)\right) \leq c \min \left\{R \log (R)\left(b_{R}-a_{R}\right), R\right\}
$$


In [25], it is shown that a wide class of fields satisfy (2.6), so this assumption is reasonable. We do not prove this result formally here because Proposition 2.12 is simpler to prove, holds for a wider class of fields, and suffices for its intended purpose (see Remark 2.24).

In light of Theorem 2.11, the fact that (2.6) is expected to hold for a wide class of fields also suggests it should be much easier to prove the differentiability of $c_{E S}$ away from zero, since the probability of four-arm saddles in $B(R)$ should decay exponentially at non-zero levels.

\subsubsection{The positivity of the level set density}

In order for Theorem 2.2 to describe the leading-order asymptotics of the number of excursion/level set components, it is crucial that the limiting constants are positive; if they are not, then it can be shown that $f$ almost surely has no compact excursion/level sets. One nice consequence of the differentiability of $c_{E S}$ and $c_{L S}$ is that it gives a new, short proof of their positivity in the delicate case $\ell=0$ :

Proposition 2.14. Let $f$ be a Gaussian field satisfying Assumption 2.1. Suppose either $c_{E S}$ or $c_{L S}$ is differentiable at 0 . Then $c_{E S}(0)>0$ and $c_{L S}(0)>0$.

The positivity of $c_{E S}(0)$ and $c_{L S}(0)$ are already known quite generally $([26,18]$ give a variety of sufficient conditions, whose union can be checked to exhaust fields satisfying Assumptions 2.1 and 2.7). We restate this result because it uses a very different method of proof; in particular, it does not rely on the 'barrier method'.

The positivity of $c_{E S}(\ell)$ and $c_{L S}(\ell)$ for $\ell>0$ is simpler to establish, even without differentiability (see [6]). On the other hand, our arguments apparently do not extend to $c_{E S}(\ell)$ for $\ell<0$ (although this case can still be treated via the 'barrier method'; see Lemma 2.23).

\subsubsection{Fields outside the 'percolation universality class'}

Although in general we expect the properties of $c_{E S}$ and $c_{L S}$ to match those of the analogous density functional $\kappa$ from percolation theory, this can fail for fields outside the 'percolation universality class'.

To demonstrate this, we consider the one non-trivial case in which $c_{E S}$ and $c_{L S}$ are explicitly known: fields with spectral measure supported on four or five points (see [6, Proposition 1.20]). In [6] it was shown that, in the 'five point case', $c_{E S}$ and $c_{L S}$ are smooth everywhere, whereas in the 'four point case', $c_{E S}$ and $c_{L S}$ are smooth everywhere except zero, at which point they are continuous but not differentiable (see Figure 2). Hence, in both cases, the smoothness of $c_{E S}$ and $c_{L S}$ differs from the conjectured properties of $\kappa$ (and in different ways). However, these fields do not fall within the scope of the present paper (they do not satisfy Assumptions 2.1 and 2.7). Moreover, being periodic, their large-scale properties cannot be expected to match those of Bernoulli percolation.

On the other hand, the non-differentiability of $c_{E S}$ at zero in the 'four point case' does reflect a different kind of phase transition: for $\ell \leq 0,\{f \geq \ell\}$ almost surely has no bounded components $\left(c_{E S}(\ell)=0\right)$, whereas for $\ell>0$, the number of components is of order $R^{2}\left(c_{E S}(\ell)>0\right)$; see Figure 3. Moreover, a Gaussian field in the 'five point case' can be represented as a field in the 'four point case' plus an independent Gaussian level shift. Hence the same phase transition occurs, although it does so at a random level and so the discontinuity is averaged out. 


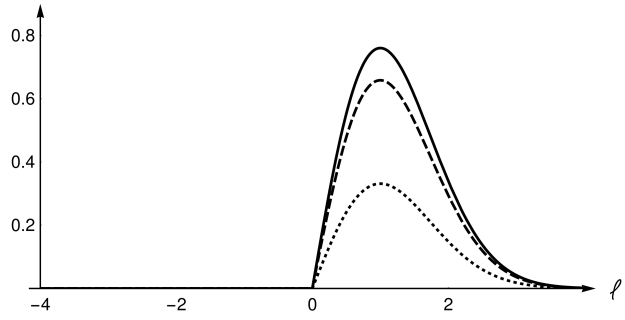

(a)

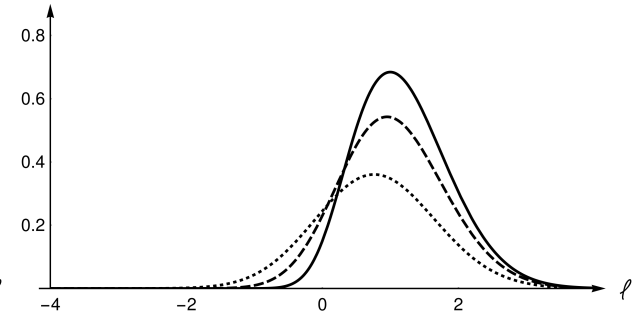

(b)

Figure 2: The functional $c_{E S}(\ell)$ for fields with spectral measure supported on four (left) or five (right) points. The different lines correspond to different measures.
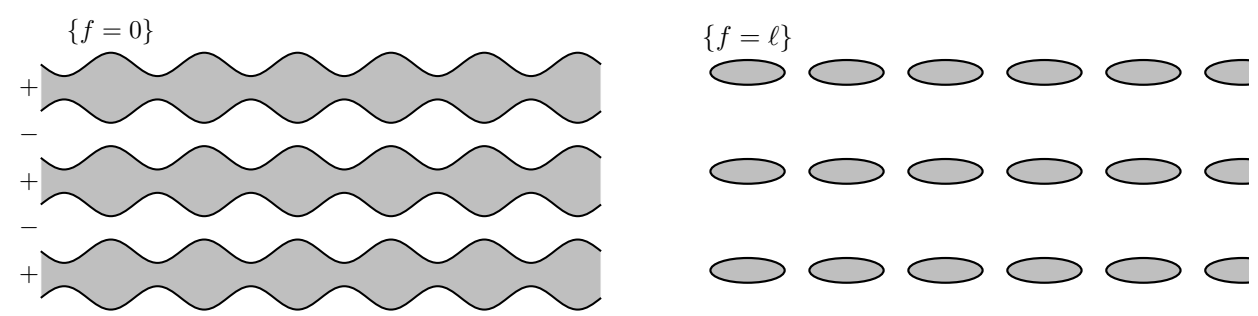

Figure 3: Stylised excursion sets for fields with spectral measure supported on four points, at the zero level (left) and at a positive level (right).

\subsection{Monotonicity}

We next consider monotonicity properties of $c_{E S}$ and $c_{L S}$. We begin by analysing the ratio

$$
p_{s^{-}}^{*}(\ell) / p_{s}(\ell)=\mathbb{P}\left(\tilde{f}_{\ell} \text { has a lower connected saddle point at the origin }\right)
$$

which we intuitively expect to be non-decreasing: if we condition on the origin being a saddle point at increasing heights, it seems more likely that it should be lower connected. This can be made rigorous under some additional assumptions, and allows us to deduce regions on which $c_{E S}(\ell)$ and $c_{L S}(\ell)$ are monotone.

Assumption 2.15. The field $f$ is isotropic (i.e. its law is invariant under rotations) and hence its covariance function can be expressed as $K(x)=k\left(|x|^{2}\right)$. Then

$$
\chi:=\frac{-k^{\prime}(0)}{\sqrt{k^{\prime \prime}(0)}} \geq 1 .
$$

Furthermore, the Gaussian vector $\left(f(0), \nabla^{2} f(0)\right)$ is non-degenerate, and for all $x \in \mathbb{R}^{2}$,

$$
\begin{aligned}
& \mathbb{E}\left(f(x) \mid f(0)=0, \nabla^{2} f(0)=\left(\begin{array}{ll}
1 & 0 \\
0 & 0
\end{array}\right)\right) \geq 0, \\
& \mathbb{E}\left(f(x) \mid f(0)=1, \nabla^{2} f(0)=\left(\begin{array}{ll}
0 & 0 \\
0 & 0
\end{array}\right)\right) \leq 1 .
\end{aligned}
$$

The parameter $\chi$ is used in [12] to parameterise the density of eigenvalues of $\nabla^{2} f$ at critical points and is shown to take values in $(0, \sqrt{2}]$. We note that $(2.7)$ can be replaced by a weaker condition (see Remark 5.8) however we do not state the general condition here as (2.7) is much simpler to verify. 
In Section 5.1.2 we explain how (2.8) and (2.9) can be translated into explicit properties of the conditional field $\tilde{f}_{\ell}$. We can also give equivalent versions of (2.8) and (2.9) that are easier to check in practice. If we rescale the domain of $f$ so that $k^{\prime}(0)=-1$ (note that this does not affect the value of $\chi$ ), then it can be shown by Gaussian regression that (2.8) is equivalent to

$$
\forall x \in \mathbb{R}^{2}, \quad\left(k\left(|x|^{2}\right)+k^{\prime}\left(|x|^{2}\right)\right) k^{\prime \prime}(0)+\left(x_{1}^{2}\left(3 k^{\prime \prime}(0)-1\right)+x_{2}^{2}\left(1-k^{\prime \prime}(0)\right)\right) k^{\prime \prime}\left(|x|^{2}\right) \geq 0
$$

and (2.9) is equivalent to

$$
\forall y \geq 0, \quad \frac{2 k^{\prime \prime}(0) k(y)+y k^{\prime \prime}(y)+k^{\prime}(y)}{2 k^{\prime \prime}(0)-1} \leq 1 .
$$

From this, it can be verified that specific fields satisfy Assumption 2.15, including the BF field. The RPW does not satisfy Assumption 2.15; in this case $\chi=\sqrt{2}$ but $\left(f(0), \nabla^{2} f(0)\right)$ is non-degenerate so the conditional expectations in (2.8) and (2.9) are not defined. However we are able to prove the monotonicity of $p_{s^{-}}^{*} / p_{s}$ in this case too:

Theorem 2.16. Let $f$ be the Random Plane Wave or a field which satisfies Assumptions 2.1, 2.7 and 2.15 (e.g. the Bargmann-Fock field). Then $p_{s^{-}}^{*}(\ell) / p_{s}(\ell)$ is non-decreasing in $\ell$.

Given the definition of $p_{s^{-}}^{*}$, we will show that Theorem 2.16 is a consequence of $\tilde{f}_{\ell}-\ell$ being stochastically decreasing in $\ell$. Our proof of the latter fact differs for the RPW and for fields satisfying Assumption 2.15 (in the former case it is somewhat simpler, because of the degeneracies in the RPW; see e.g. [33]).

The monotonicity of $p_{s^{-}}^{*} / p_{s}$ has some implications for the smoothness of $c_{E S}$ and $c_{L S}$ :

Corollary 2.17. Let $f$ satisfy the conditions of Theorem 2.16, then $p_{s^{-}}^{*}$ has at most a countable set of discontinuities, all of which are jump discontinuities. In particular, $c_{E S}$ and $c_{L S}$ are twice differentiable almost everywhere.

Another consequence of monotonicity is a converse of Theorem 2.11:

Corollary 2.18. Let $f$ satisfy the conditions of Theorem 2.16, then for every $a<b$ the following are equivalent:

1. For all $\ell \in(a, b)$

$$
\mathbb{P}\left(\tilde{f}_{\ell} \text { has an infinite four-arm saddle at the origin }\right)=0 ;
$$

2. There exists a version of $p_{s^{-}}$which is continuous on $(a, b)$;

3. $c_{E S}(\cdot)$ is continuously differentiable on $(a, b)$;

4. $c_{L S}(\cdot)$ is continuously differentiable on $(a, b)$.

Remark 2.19. Clearly, if any of (1)-(4) hold in Corollary 2.18, then by Theorem 2.11, the version of $\left.p_{s^{-}}\right|_{(a, b)}$ which is continuous is equal to $\left.p_{s^{-}}^{*}\right|_{(a, b)}$.

Finally we use Theorem 2.16 to deduce intervals on which $c_{E S}$ and $c_{L S}$ are monotone. We shall state the strongest form of our results only in the case of the RPW and BF field. Let $D_{+}$and $D^{+}$respectively denote the lower and upper, right Dini derivatives, that is, for $g: \mathbb{R} \rightarrow \mathbb{R}$,

$$
D_{+} g(x)=\liminf _{\epsilon \rightarrow 0^{+}} \frac{g(x+\epsilon)-g(x)}{\epsilon} \text { and } D^{+} g(x)=\limsup _{\epsilon \rightarrow 0^{+}} \frac{g(x+\epsilon)-g(x)}{\epsilon} .
$$

Proposition 2.20. Let $f$ be the Random Plane Wave. Then

$$
\begin{array}{ll}
D_{+} c_{E S}(\ell)>0 & \text { for } \ell \in(-\infty, 0.87] \\
D^{+} c_{E S}(\ell)<0 & \text { for } \ell \in[1, \infty)
\end{array}
$$


Smoothness/monotonicity of the excursion set density of Gaussian fields

and

$$
D^{+} c_{L S}(\ell)<0 \text { for } \ell \in[1, \infty) \text {. }
$$

Proposition 2.21. Let $f$ be the Bargmann-Fock field. Then there exists $\epsilon>0$ such that

$$
c_{E S}^{\prime}(\ell) \begin{cases}>0 & \text { for } \ell \in(-\epsilon, 0.64] \\ <0 & \text { for } \ell \in[1.03, \infty)\end{cases}
$$

and

$$
c_{L S}^{\prime}(\ell)<0 \text { for } \ell \in[1.03, \infty)
$$

We also present weaker results for general isotropic fields. Recall that the covariance function of an isotropic $f$ may be expressed as $K(x)=k\left(|x|^{2}\right)$ for some $k:[0, \infty) \rightarrow \mathbb{R}$. We also recall the parameter $\chi=-k^{\prime}(0) / \sqrt{k^{\prime \prime}(0)}$ which takes values in $(0, \sqrt{2}]$ (see [12] for details on this parameter).

Proposition 2.22. Let $f$ be an isotropic field which satisfies Assumptions 2.1, 2.8-2.9 and 2.15. Then there exists $\epsilon>0$ and an explicit constant $C>0$ such that

$$
c_{E S}^{\prime}(\ell) \begin{cases}>0 & \text { for } \ell \in(-\epsilon, C) \\ <0 & \text { for } \ell \in(\sqrt{2} / \chi, \infty)\end{cases}
$$

and

$$
c_{L S}^{\prime}(\ell)<0 \text { for } \ell \in(\sqrt{2} / \chi, \infty) .
$$

The explicit formula for the constant $C$ is quite complicated and is given in the proof of this proposition. However it is straightforward to apply this formula to any particular field (as we have done for the RPW and Bargmann-Fock field in Propositions 2.20 and 2.21).

As an intermediate result to Proposition 2.20 we require that, for the RPW, $c_{E S}(\ell)>0$ for $\ell \leq 0$. Since this result is not stated elsewhere in the literature, we do so here. The proof uses the 'barrier method' and is near-identical to that in [26] in the case $\ell=0$.

Proposition 2.23. Let $f$ be the Random Plane Wave. Then $c_{E S}(\ell)>0$ for all $\ell \in \mathbb{R}$.

Remark 2.24. Many of the results in this work are built upon by [5] in order to prove lower bounds on the variance of the number of level/excursion set components in $B(R)$ as $R \rightarrow \infty$. Specifically, it is shown that if $f$ has sufficiently nice correlation decay (such as the BF field), and if $c_{E S}$ has a non-zero derivative at $\ell$, then

$$
\operatorname{Var}\left(N_{E S}(R, \ell)\right) \geq c R^{2}
$$

for some $c>0$ and all $R$ sufficiently large. Moreover, if $f$ is the RPW and one of the Dini derivatives of $c_{E S}$ is non-zero for $\ell \neq 0$, then

$$
\operatorname{Var}\left(N_{E S}(R, \ell)\right) \geq c R^{3}
$$

for some $c>0$ and all $R$ sufficiently large. Analogous results hold in both cases for level sets and $c_{L S}$. A key step in proving these results is to estimate the order of

$$
\mathbb{E}\left(N_{E S}(R, \ell)-N_{E S}\left(R, \ell+\epsilon_{R}\right)\right),
$$

which is made possible by Theorem 2.10 and Corollary 2.12. Since the lower bounds also require that $c_{E S}$ has a non-zero derivative/Dini derivative at $\ell$, Propositions 2.20-2.22 are crucial for ensuring that they are widely applicable. 


\subsection{Outline of the remainder of the paper}

In Section 3 we give a formal definition of $\tilde{f}_{\ell}$, the field $f$ conditioned to have a saddle point at the origin at level $\ell$, and derive explicit representations for $\tilde{f}_{\ell}$ in special cases. In Section 4 we study topological properties of $\tilde{f}_{\ell}$, and use this to deduce the results outlined in Section 2.1. In Section 5 we consider stochastic monotonicity properties of $\tilde{f}_{\ell}$, and complete the proofs of the results in Section 2.2. Appendix A contains miscellaneous results on the non-degeneracy of Gaussian fields.

\section{The field conditioned to have a saddle at the origin}

In this section we consider $\tilde{f}_{\ell}$, the field $f$ conditioned to have a saddle point at the origin at level $\ell$. Using the theory of Palm distributions we give an explicit representation for $\tilde{f}_{\ell}$, and in the isotropic case we derive simple expressions for its distribution.

We begin with a general statement expressing $\tilde{f}_{\ell}$ as (a limit of) a Palm distribution relative to a point process defined by the saddle points of $f$. Let us first recall the relevant theory of Palm distributions (see [21, Chapter 11] for background). We define a point process $\zeta$ to be a random measure on $\mathbb{R}^{d}$ such that $\zeta(B)$ is integer-valued for every bounded Borel set $B$. We say that $\zeta$ is simple if, with probability one, $\zeta(\{s\}) \leq 1$ for every $s \in \mathbb{R}^{d}$. We say that it is non-degenerate if $\mathbb{E}(\zeta(B))>0$ for every Borel set $B$ with positive Lebesgue measure. Let $g: \mathbb{R}^{2} \rightarrow \mathbb{R}$ be a planar random field and $\mathcal{S}$ a non-degenerate, simple point process on $\mathbb{R}^{2}$, and suppose that $(g, \mathcal{S})$ are jointly stationary (i.e. this joint distribution is invariant under translations). Fix a bounded Borel set $B \subset \mathbb{R}^{2}$ such that $0<\mathbb{E}(\#\{s \in B: s \in \mathcal{S}\})<\infty$. Then the Palm distribution of $g$ relative to $\mathcal{S}$ is defined as the random field $\tilde{g}$ satisfying, for any Borel cylinder set $A$,

$$
\mathbb{P}(\tilde{g}(x) \in A)=\frac{\mathbb{E}(\#\{s \in B: s \in \mathcal{S}, g(x-s) \in A\})}{\mathbb{E}(\#\{s \in B: s \in \mathcal{S}\})} .
$$

This definition is independent of the reference set $B$, and we may therefore write $\tilde{g}=(g \mid\{0\} \in \mathcal{S})$.

Lemma 3.1. Let $f$ be a Gaussian field satisfying Assumption 2.1. For $\ell \in \mathbb{R}$ and $\epsilon>0$, let

$$
\tilde{f}_{[\ell, \ell+\epsilon]}=(f \mid\{0\} \in S[\ell, \ell+\epsilon])
$$

be the Palm distribution of $f$ relative to $S[\ell, \ell+\epsilon]$, the point process of saddle points with level in $[\ell, \ell+\epsilon]$ (this point process is non-degenerate by Lemma A.3). Then there exists a random field $\tilde{f}_{\ell}$ such that, as $\epsilon \rightarrow 0, \tilde{f}_{[\ell, \ell+\epsilon]}$ converges in distribution to $\tilde{f}_{\ell}$ in the topology of uniform $C^{2+\eta}$ convergence on compacts.

It is important to distinguish $\tilde{f}_{[\ell, \ell+\epsilon]}$ from the conditioned field

$$
\left(f(t) \mid \nabla f(0)=0, \operatorname{det} \nabla^{2} f(0)<0, f(0) \in[\ell, \ell+\epsilon]\right),
$$

which is defined via the distributional limit

$$
\lim _{\delta \rightarrow 0}\left(f(t) \mid f_{1}(0), f_{2}(0) \in[0, \delta), \operatorname{det} \nabla^{2} f(0)<0, f(0) \in[\ell, \ell+\epsilon]\right) .
$$

The latter is sometimes known as 'vertical window conditioning', and is the standard way of conditioning on part of a random vector (for a Gaussian vector, this conditioning is given explicitly by Gaussian regression, see [3, Proposition 1.2]). By constrast, the former can be thought of as 'horizontal window conditioning', and corresponds to sampling a 'typical' saddle point (i.e. via the counting measure). The difference between these forms of conditioning is elegantly explained in [20].

Using basic properties of Gaussian fields, we can derive explicit representations for $\tilde{f}_{[\ell, \ell+\epsilon]}$ and $\tilde{f}_{\ell}$ : 
Lemma 3.2. Let $f$ be a Gaussian field satisfying Assumption 2.1 such that $\left(f(0), \nabla^{2} f(0)\right)$ is a non-degenerate Gaussian vector. Define $\alpha: \mathbb{R}^{2} \rightarrow \mathbb{R}$ and $\beta=\left(\beta_{11}, \beta_{22}, \beta_{12}\right): \mathbb{R}^{2} \rightarrow \mathbb{R}^{3}$ to be the unique functions satisfying

$$
\mathbb{E}\left(f(t) \mid f(0)=u, \nabla^{2} f(0)=U\right)=\alpha(t) u+\beta(t) \cdot U
$$

for all $u \in \mathbb{R}, U \in \mathbb{R}^{3}$, and define

$$
\gamma(s, t):=\mathbb{E}\left(f(s) f(t) \mid f(0)=0, \nabla f(0)=0, \nabla^{2} f(0)=0\right) .
$$

Then

$$
\tilde{f}_{[\ell, \ell+\epsilon]} \stackrel{d}{=} g+z_{[\ell, \ell+\epsilon]} \alpha+Z_{[\ell, \ell+\epsilon]} \cdot \beta,
$$

where $g$ is a centred Gaussian field with covariance function $\gamma$, and $\left(z_{[\ell, \ell+\epsilon]}, Z_{[\ell, \ell+\epsilon]}\right)$ is an independent random vector with density ${ }^{1}$

$$
p_{\left(z_{[\ell, \ell+\epsilon]}, Z_{[\ell, \ell+\epsilon]}\right)}(x, X) \propto|\operatorname{det} X| p_{f(0), \nabla^{2} f(0)}(x, X) \mathbb{1}_{x \in[\ell, \ell+\epsilon]} \mathbb{1}_{\operatorname{det} X<0}
$$

Moreover,

$$
\tilde{f}_{\ell} \stackrel{d}{=} g+\ell \alpha+Z_{\ell} \cdot \beta
$$

where $Z_{\ell}$ is a random vector, independent of $g$, with density

$$
p_{Z_{\ell}}(X) \propto|\operatorname{det} X| p_{f(0), \nabla^{2} f(0)}(\ell, X) \mathbb{1}_{\operatorname{det} X<0}
$$

The functions $\alpha, \beta$ and $\gamma$ in Lemma 3.2 can be computed explicitly via Gaussian regression (see [3, Proposition 1.2]). Specifically, define $v_{0}=\left(f(0), \partial_{x x} f(0), \partial_{y y} f(0), \partial_{x y} f(0)\right)$ and

$$
v=\left(f(0), \nabla f(0), \partial_{x x} f(0), \partial_{y y} f(0), \partial_{x y} f(0)\right),
$$

and let $\Sigma_{0}$ and $\Sigma$ be the respective covariance matrices of these vectors. Then

$$
\left(\alpha(t), \beta_{11}(t), \beta_{22}(t), \beta_{12}(t)\right)=\operatorname{Cov}\left(f(t), v_{0}\right) \Sigma_{0}^{-1}
$$

and

$$
\gamma(s, t)=\operatorname{Cov}(f(s), f(t))-\operatorname{Cov}(f(s), v) \Sigma^{-1} \operatorname{Cov}(f(t), v)^{\prime} .
$$

In the case that $\left(f(0), \nabla^{2} f(0)\right)$ is degenerate (which includes the RPW), the representations of $\tilde{f}_{[\ell, \ell+\epsilon]}$ and $\tilde{f}_{\ell}$ in Lemma 3.2 must be modified to accommodate this degeneracy; in particular, $\nabla^{2} f(0)$ should be considered as a vector consisting of two of its coordinates, chosen so that they are non-degenerate with $f(0)$, and $\alpha, \beta$ and $\gamma$ defined accordingly. For simplicity we will not state this representation formally; for the RPW we state a more precise description below (in Proposition 3.4).

Lemmas 3.1 and 3.2 are essentially derived in [2, Chapter 6]; we repeat this here for completeness, and so that we can extend the arguments slightly.

Proof of Lemmas 3.1 and 3.2. We assume that $\left(f(0), \nabla^{2} f(0)\right)$ is non-degenerate, since the proof in the degenerate case is almost identical. Let $T=\left(t_{1}, \ldots, t_{m}\right) \in \mathbb{R}^{2 m}$ and

\footnotetext{
${ }^{1}$ Here and in the proof of this lemma we treat $Z_{[\ell, \ell+\epsilon]}$ interchangeably as the three-dimensional column $\operatorname{vector}\left(Z_{[\ell, \ell+\epsilon], 11}, Z_{[\ell, \ell+\epsilon], 22}, Z_{[\ell, \ell+\epsilon], 12}\right)$ and the symmetric $2 \times 2$ matrix $\left(\begin{array}{c}Z_{[\ell, \ell+\epsilon], 11} Z_{[\ell, \ell+\epsilon], 12} \\ Z_{[\ell, \ell+\epsilon], 12} Z_{[\ell, \ell+\epsilon], 22}\end{array}\right)$; which form we are using will always be clear from context. We also use this convention for $Z_{\ell}$ and $X$ (introduced below).
} 
$y_{1}, \ldots, y_{m} \in \mathbb{R}$. Then by the definition of $\tilde{f}_{[\ell, \ell+\epsilon]}$, and the Kac-Rice theorem ([1, Corollary 11.2.2]),

$$
\begin{aligned}
& \mathbb{P}\left(\tilde{f}_{[\ell, \ell+\epsilon]}\left(t_{1}\right) \leq y_{1}, \ldots, \tilde{f}_{[\ell, \ell+\epsilon]}\left(t_{m}\right) \leq y_{m}\right) \\
& =\frac{\mathbb{E}\left(\#\left\{s \in B(1): \nabla f(s)=0, \operatorname{det} \nabla^{2} f(s)<0, f(s) \in[\ell, \ell+\epsilon], f\left(s+t_{i}\right) \leq y_{i} \forall i\right)\right.}{\mathbb{E}\left(\#\left\{s \in B(1): \nabla f(s)=0, \operatorname{det} \nabla^{2} f(s)<0, f(s) \in[\ell, \ell+\epsilon]\right)\right.} \\
& =\frac{\mathbb{E}\left(\left|\operatorname{det} \nabla^{2} f(0)\right| \mathbb{1}_{f(0) \in[\ell, \ell+\epsilon]} \mathbb{1}_{\operatorname{det} \nabla^{2} f(0)<0} \prod_{i=1}^{m} \mathbb{1}_{\left.f\left(t_{i}\right) \leq y_{i} \mid \nabla f(0)=0\right)}\right.}{\mathbb{E}\left(\left|\operatorname{det} \nabla^{2} f(0)\right| \mathbb{1}_{f(0) \in[\ell, \ell+\epsilon]} \mathbb{1}_{\operatorname{det} \nabla^{2} f(0)<0} \mid \nabla f(0)=0\right)} \\
& =\int_{-\infty}^{y_{1}} \cdots \int_{-\infty}^{y_{m}} \frac{\int_{\mathbb{R}^{3}} \int_{\ell}^{\ell+\epsilon}|\operatorname{det} X| p_{T}(x, 0, X, U) \mathbb{1}_{\operatorname{det} X<0} d x d X}{\int_{\mathbb{R}^{3}} \int_{\ell}^{\ell+\epsilon}|\operatorname{det} X| p(x, 0, X) \mathbb{1}_{\operatorname{det} X<0} d x d X} d U_{m} \ldots d U_{1},
\end{aligned}
$$

where $p_{T}$ and $p$ denote respectively the densities of $\left(f(0), \nabla f(0), \nabla^{2} f(0), f\left(t_{1}\right), \ldots, f\left(t_{m}\right)\right)$ and $\left(f(0), \nabla f(0), \nabla^{2} f(0)\right)$. We note that $p$ is non-degenerate since $\nabla f(0)$ is independent of $\left(f(0), \nabla^{2} f(0)\right)$ (this is a standard fact for Gaussian fields with constant variance, see $\left[1\right.$, Chapter 5]) and these vectors are non-degenerate by assumption. The density $p_{T}$ may be degenerate, in which case we think of it as having atomic mass. Rearranging these terms slightly, we can express the joint density of $\left(\tilde{f}_{[\ell, \ell+\epsilon]}\left(t_{i}\right): i=1, \ldots, m\right)$ as

$$
\varphi_{T}^{[\ell, \ell+\epsilon]}(U):=\int_{\mathbb{R}^{3}} \int_{\ell}^{\ell+\epsilon} \psi_{x}(X) p_{T}(x, 0, X, U) / p(x, 0, X) d x d X
$$

where

$$
\psi_{x}(X)=\frac{|\operatorname{det} X| p(x, 0, X) \mathbb{1}_{\operatorname{det} X<0}}{\int_{\mathbb{R}^{3}} \int_{\ell}^{\ell+\epsilon}|\operatorname{det} X| p(x, 0, X) \mathbb{1}_{\operatorname{det} X<0} d x d X} .
$$

Then the characteristic function of $\left(\tilde{f}_{[\ell, \ell+\epsilon]}\left(t_{1}\right), \ldots, \tilde{f}_{[\ell, \ell+\epsilon]}\left(t_{m}\right)\right)$ is given by

$$
\hat{\varphi}_{T}^{[\ell, \ell+\epsilon]}(\theta)=\int_{\mathbb{R}^{3}} \int_{\ell}^{\ell+\epsilon} \psi_{x}(X) \int_{\mathbb{R}^{m}} e^{i \theta \cdot U} p_{T}(x, 0, X, U) / p(x, 0, X) d U d x d X .
$$

The inner integral of equation (3.2) can be calculated by Gaussian regression (see [3, Proposition 1.2]). Specifically, let $A=\left(\alpha\left(t_{1}\right), \ldots, \alpha\left(t_{m}\right)\right)^{\prime}, B=\left(\beta\left(t_{1}\right), \ldots, \beta\left(t_{m}\right)\right)^{\prime}$ and $\Gamma=\left(\gamma\left(t_{i}, t_{j}\right)\right)_{i, j=1, \ldots, m}$, then

$$
\left(f\left(t_{1}\right), \ldots, f\left(t_{m}\right) \mid f(0)=x, \nabla f(0)=0, \nabla^{2} f(0)=X\right) \sim \mathcal{N}(A x+B X, \Gamma) .
$$

Since $p_{T}(x, 0, X, U) / p(x, 0, X)$ is the probability density of this random variable, we can substitute the characteristic function of a Gaussian vector into (3.2) to give

$$
\begin{aligned}
\hat{\varphi}_{T}^{[\ell, \ell+\epsilon]}(\theta) & =\int_{\mathbb{R}^{3}} \int_{\ell}^{\ell+\epsilon} \psi_{x}(X) e^{i \theta \cdot(A x+B X)-\frac{1}{2} \theta^{\prime} \Gamma \theta} d x d X \\
& =e^{-\frac{1}{2} \theta^{\prime} \Gamma \theta} \frac{\int_{\mathbb{R}^{4}} e^{i \theta \cdot(A x+B X)}|\operatorname{det} X| p(x, 0, X) \mathbb{1}_{x \in[\ell, \ell+\epsilon]} \mathbb{1}_{\operatorname{det} X<0} d x d X}{\int_{\mathbb{R}^{4}}|\operatorname{det} X| p(x, 0, X) \mathbb{1}_{x \in[\ell, \ell+\epsilon]} \mathbb{1}_{\operatorname{det} X<0} d x d X} .
\end{aligned}
$$

Since the characteristic function of a random vector uniquely specifies its distribution, we identify the distribution of $\tilde{f}_{[\ell, \ell+\epsilon]}$ as that given in the statement of Lemma 3.2 (using the fact that $p(x, 0, X)=p_{f(0), \nabla^{2} f(0)}(x, X)$ since $\nabla f(0)$ is independent of $\left.\left(f(0), \nabla^{2} f(0)\right)\right)$.

By inspecting their joint distribution, it is clear that $z_{[\ell, \ell+\epsilon]} \stackrel{d}{\rightarrow} \ell$ and $Z_{[\ell, \ell+\epsilon]} \stackrel{d}{\rightarrow} Z_{\ell}$ as $\epsilon \rightarrow 0$. We now fix a sequence $\epsilon_{i} \downarrow 0$, and create a coupling of $\tilde{f}_{\left[\ell, \ell+\epsilon_{i}\right]}$ for each $i$ such that each field consists of the same realisation of $g$ and the sequences $\left\{z_{\left[\ell, \ell+\epsilon_{i}\right]}\right\}_{i \in \mathbb{N}}$ and $\left\{Z_{\left[\ell, \ell+\epsilon_{i}\right]}\right\}_{i \in \mathbb{N}}$ converge almost surely. Since $K \in C_{\text {loc }}^{4+\eta^{\prime}}\left(\mathbb{R}^{2}\right)$, the same is true of $\alpha, \beta$ 
and $\gamma$ and hence $g \in C_{\operatorname{loc}}^{2+\eta}\left(\mathbb{R}^{2}\right)$ almost surely for the choice of $\eta \in\left(0, \eta^{\prime} / 2\right)$ made at the beginning of Section 2 (Kolmogorov's theorem [26, Appendix A]). It is therefore clear that the coupled fields $\tilde{f}_{\left[\ell, \ell+\epsilon_{i}\right]}$ converge almost surely in the $C^{2+\eta}$ topology uniformly on compact sets as $i \rightarrow \infty$ to $\tilde{f}_{\ell}$. This completes the proof of the lemmas.

We now present simpler descriptions for $\tilde{f}_{\ell}$ in the case of isotropic fields. In this case it is quite natural to express the Hessian component $Z_{\ell}$ in terms of its eigenvalues $\lambda_{1}<\lambda_{2}$ and the argument $\theta$ of the first eigenvector. Recall the parameter $\chi=-k^{\prime}(0) / \sqrt{k^{\prime \prime}(0)} \in$ $(0, \sqrt{2}]$, where $K(t)=k\left(|t|^{2}\right)$. Again we must distinguish the case in which $\left(f(0), \nabla^{2} f(0)\right)$ is degenerate, which corresponds to $\chi=\sqrt{2}$ and implies that $f$ is (a rescaled version of) the RPW.

Proposition 3.3. Let $f$ be an isotropic field satisfying Assumption 2.1 such that $\chi<\sqrt{2}$. Then

$$
\tilde{f}_{\ell}(\cdot) \stackrel{d}{=} g(\cdot)+\ell \alpha(\cdot)+\lambda_{1} b_{1}(\cdot, \theta)+\lambda_{2} b_{2}(\cdot, \theta)
$$

where $g, \alpha$ and $\beta$ are as in Lemma 3.2,

$$
\begin{aligned}
& b_{1}(t, \theta)=\cos ^{2}(\theta) \beta_{11}(t)+\sin ^{2}(\theta) \beta_{22}(t)+\sin (\theta) \cos (\theta) \beta_{12}(t), \\
& b_{2}(t, \theta)=\sin ^{2}(\theta) \beta_{11}(t)+\cos ^{2}(\theta) \beta_{22}(t)+\sin (\theta) \cos (\theta) \beta_{12}(t),
\end{aligned}
$$

$\theta$ is an independent random variable uniform on $[0,2 \pi)$, and $\left(\lambda_{1}, \lambda_{2}\right)$ is an independent random vector with density proportional to

$$
q_{\ell}(x, y):=|x| y(y-x) \mathbb{1}_{y>0>x} \exp \left(-\frac{1}{2 \sigma^{2}}\left((x-\mu \ell)^{2}+(y-\mu \ell)^{2}+2 \tau(x-\mu \ell)(y-\mu \ell)\right)\right)
$$

where

$$
\mu=2 k^{\prime}(0), \quad \sigma^{2}=\frac{16 k^{\prime \prime}(0)\left(2-\chi^{2}\right)}{3-\chi^{2}} \quad \text { and } \quad \tau=\frac{\chi^{2}-1}{3-\chi^{2}}
$$

Proof. Recall the random vector $Z_{\ell}$ from Lemma 3.2, which we view as a $2 \times 2$ symmetric matrix. Let $\lambda_{1}<\lambda_{2}$ be the eigenvalues of $Z_{\ell}$, and let $\theta$ be the argument of the eigenvector associated to $\lambda_{1}$. If $h$ denotes the bijection which maps $Z_{\ell}$ to $\Lambda:=\left(\lambda_{1}, \lambda_{2}, \theta\right)$, then for any Borel set $A$

$$
\mathbb{P}(\Lambda \in h(A))=\frac{\mathbb{E}\left(\left|\operatorname{det} \nabla^{2} f(0)\right| \mathbb{1}_{\operatorname{det} \nabla^{2} f(0)<0, \nabla^{2} f(0) \in A} \mid f(0)=\ell\right)}{\mathbb{E}\left(\left|\operatorname{det} \nabla^{2} f(0)\right| \mathbb{1}_{\operatorname{det} \nabla^{2} f(0)<0} \mid f(0)=\ell\right)} .
$$

Since $f$ is isotropic and $\left(f(0), \nabla^{2} f(0)\right)$ is non-degenerate, [12] derives the density of the ordered eigenvalues of $\left(\nabla^{2} f(0) \mid f(0)=\ell\right)$ and the argument of the corresponding eigenvectors as that given above.

Proposition 3.4. Let $f$ be the Random Plane Wave. Then

$$
\tilde{f}_{\ell} \stackrel{d}{=} g+\ell \alpha+Z^{\ell} \cdot \beta
$$

where $g$ is a centred Gaussian field with covariance function $\gamma$ (defined as in Lemma 3.2), $\alpha, \beta$ are defined as

$$
\begin{gathered}
\alpha(t)=J_{0}(|t|)+2 \frac{t_{1}^{2}-t_{2}^{2}}{|t|^{2}} J_{2}(|t|), \\
\beta_{11}(t)=4 \frac{t_{1}^{2}-t_{2}^{2}}{|t|^{2}} J_{2}(|t|), \quad \beta_{12}(t)=8 \frac{t_{1} t_{2}}{|t|^{2}} J_{2}(|t|),
\end{gathered}
$$


and $Z^{\ell}=\left(Z_{11}^{\ell}, Z_{12}^{\ell}\right)^{t}$ is an independent random vector with density

$$
\psi_{\ell}(x, y) \propto\left(x(x+\ell)+y^{2}\right) \mathbb{1}_{\left(x(x+\ell)+y^{2}\right)>0} p_{f(0), f_{11}(0), f_{12}(0)}(\ell, x, y) .
$$

Alternatively, $\tilde{f}_{\ell}$ has the representation

$$
\tilde{f}_{\ell}(t)=g(t)+\ell \cdot\left[J_{0}(|t|)+2 \cos (2(\theta-\arg t)) J_{2}(|t|)\right]+\lambda \cdot 4 \cos (2(\theta-\arg t)) J_{2}(|t|),
$$

where $\arg t$ denotes the argument of $t$ and $(\theta, \lambda)=\left(\theta, \lambda_{\ell}\right)$ is a random vector, independent of $g$, with density

$$
p_{\lambda_{\ell}, \theta}(x, y) \propto x(x+\ell)(2 x+\ell) e^{-4 x(x+\ell)} \mathbb{1}_{x>\max \{0,-\ell\}} \mathbb{1}_{y \in[0,2 \pi)} .
$$

Proof. The representation

$$
\tilde{f}_{\ell}=g+\ell \alpha+Z^{\ell} \cdot \beta
$$

follows from an argument similar to that used to prove Lemma 3.2. The functions $\alpha$ and $\beta$ can be explicitly calculated using Gaussian regression (see [3, Proposition 1.2] for example). Next we note that $\left(Z_{11}^{\ell}, Z_{12}^{\ell}\right)$ is supported on the region for which

$$
\operatorname{det}\left(\begin{array}{cc}
Z_{11}^{\ell} & Z_{12}^{\ell} \\
Z_{12}^{\ell} & -Z_{11}^{\ell}-\ell .
\end{array}\right)<0 .
$$

Therefore this matrix almost surely has a unique, positive eigenvalue $\lambda$ and corresponding eigenvector with argument $\theta$. By explicitly diagonalising this matrix, we obtain a formula for $\lambda$ and $\theta$ :

$$
\begin{aligned}
Z_{11}^{\ell}+\ell / 2 & =(\lambda+\ell / 2) \cos (2 \theta) \\
Z_{12}^{\ell} & =(\lambda+\ell / 2) \sin (2 \theta) .
\end{aligned}
$$

By the standard change of variable formula (and explicitly evaluating $\psi_{\ell}$ in terms of the covariance of the RPW) we can calculate the joint density of $(\lambda, \theta)$ to be equal to the expression in (3.4).

\section{Differentiability of excursion/level set functionals}

In this section we prove the results stated in Section 2.1. We begin by studying the space $C_{\mathrm{Reg}}^{2+\eta}$ of functions $h \in C_{\mathrm{loc}}^{2+\eta}\left(\mathbb{R}^{2}\right)$ which have a non-degenerate critical point at the origin and no other critical points at level $h(0)$. We will also use the space $C_{\mathrm{Reg}}^{2+\eta}(R)$ which is the set of all $h \in C_{\text {Reg }}^{2+\eta}$ such that $h(0)$ is not a critical level of $\left.h\right|_{\partial B(R)}$. We endow these spaces with the $C_{\text {loc }}^{2+\eta}$ topology.

By showing that $\tilde{f}_{\ell} \in C_{\mathrm{Reg}}^{2+\eta}(R)$ almost surely, we prove that $\tilde{f}_{\ell}$ having an upper (or lower) connected saddle point in a compact region is a continuity event, from this we deduce Theorem 2.11 (with the other results following as consequences).

Lemma 4.1. If $h \in C_{\text {Reg }}^{2+\eta}$ has a saddle point at the origin, then this saddle point is either upper connected, lower connected or an infinite four-arm saddle.

Proof. For a small enough neighbourhood $B$ of the origin, the level set $\{h=h(0)\}$ in $B \backslash\{0\}$ consists of four curves that connect 0 to $\partial B$ (the Morse lemma [24, Lemma 2.2]). If the connected components of these curves in $\mathbb{R}^{2} \backslash B$ are all unbounded, the saddle point must be infinite four-arm. If one of them is finite, then by the implicit function theorem it is a simple $C^{1}$ curve joining two points on $\partial B$. Hence the saddle point is either upper connected (if the field takes values larger than $h(0)$ on the outer boundary of the loop) or lower connected (if the field takes values smaller the $h(0)$ on the outer boundary of the loop). 
We now consider saddle points which are upper or lower connected in a compact domain. Specifically, for a $C^{2}$ function $h$ with a saddle point $x_{0}$ we say that $x_{0}$ is $R$-lower connected if it is in the closure of only one component of $\left\{x \in B\left(x_{0}, R\right): h(x)<h\left(x_{0}\right)\right\}$. We make an analogous definition for $R$-upper connected saddles.

Lemma 4.2. Let $s^{-}(R)$ be the subset of functions $h \in C_{R e g}^{2+\eta}(R)$ such that the origin is an $R$-lower connected saddle point of $h$, then $s^{-}(R)$ is open and closed in $C_{\text {Reg }}^{2+\eta}(R)$. The same is true for the set $s^{+}(R)$ of functions with $R$-upper connected saddle points.

Proof. Let $h \in C_{\mathrm{Reg}}^{2+\eta}(R)$ have a saddle point at the origin which is $R$-lower connected; we will find a neighbourhood around $h$ which contains only functions with such saddle points at the origin. First we choose $r \in(0,1)$ sufficiently small that $h$ has a four-arm saddle in $B(r)$. Since the origin is a non-degenerate saddle point for $h, \nabla^{2} h(0)$ has eigenvalues $\lambda_{1}<0<\lambda_{2}$ and corresponding eigenvectors $v_{1}, v_{2}$. We now choose a neighbourhood $N_{1} \subset C_{\mathrm{Reg}}^{2+\eta}(R)$ of $h$ (in the topology of uniform $C^{2+\eta}$ convergence) such that for all $g \in N_{1}$,

$$
\partial_{v_{1}, v_{1}} g(0)<\lambda_{1} / 2 \text { and } \partial_{v_{2}, v_{2}} g(0)>\lambda_{2} / 2 \text {. }
$$

This ensures that each function in $N_{1}$ also has a saddle point at the origin.

Next we choose $N_{2} \subset C_{\text {Reg }}^{2+\eta}(R)$ such that for each $g \in N_{2}$,

$$
\|g\|_{C^{2+\eta}(B(R))} \leq 2\|h\|_{C^{2+\eta}(B(R))} .
$$

We consider the four line segments joining 0 to $\partial B(r)$ parallel to $v_{1}$ and $v_{2}$ and we reduce $r$ relative to $\|h\|_{C^{2+\eta}(B(R))}$ so that for each $g \in N_{1} \cap N_{2}$, the directional derivative of $g$ on this line segment (parallel to the line segment) has constant sign. This ensures that for each such $g$ the saddle point at the origin is four-arm in $B(r)$.

There exist two connected subsets $A_{1}, A_{2}$ of $\partial B(r)$ such that $h<h(0)-3 \epsilon$ on $A_{1} \cup A_{2}$ for some $\epsilon>0$ and $A_{1}$ and $A_{2}$ are in different components of $\overline{B(r)} \cap\{h<h(0)\}$ (see Figure 4). We next choose a neighbourhood $N_{3} \subset C_{\text {Reg }}^{2+\eta}(R)$ of $h$ such that $A_{1}$ and $A_{2}$ have the same properties for any function $g \in N_{3}$, with $3 \epsilon$ replaced by $2 \epsilon$, and $|g(0)-h(0)|<\epsilon$.

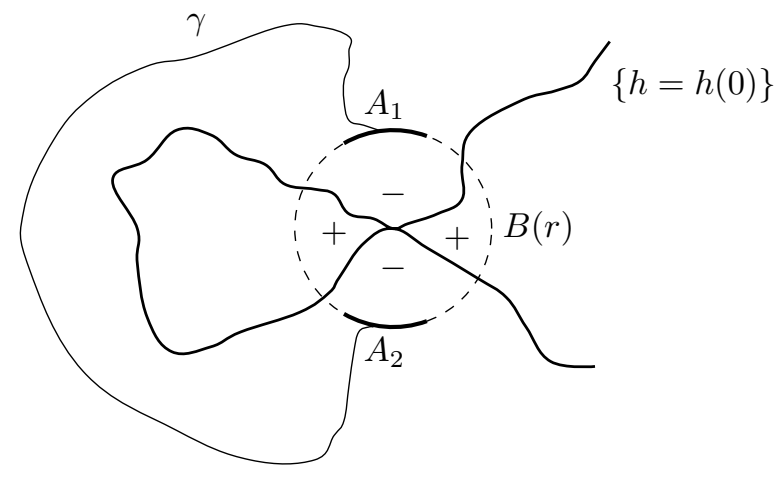

Figure 4: Approximating an $R$-lower connected saddle point in the $C^{2+\eta}(\overline{B(R)})$ topology.

By definition of a saddle being $R$-lower connected, there is a curve $\gamma$ in $B(R)$ joining $A_{1}$ to $A_{2}$ in $\{h<h(0)\}$ and $h$ is bounded above by $h(0)-3 \delta$ on $\gamma$ for some $\delta>0$. Since $\gamma$ is compact we can find a neighbourhood $N_{4}$ such that $g<h(0)-2 \delta$ on $\gamma$ for all $g \in N_{4}$ and $|h(0)-g(0)|<\delta$. Combining these observation, we see that $N:=N_{1} \cap N_{2} \cap N_{3} \cap N_{4}$ is a neighbourhood of $h$ (in $C_{\mathrm{Reg}}^{2+\eta}(R)$ ) and any $g \in N$ has a saddle point at the origin which is lower connected in $B(R)$ and so the set of functions with such saddle points is open, as required. 
The set $C_{\mathrm{Reg}}^{2+\eta}$ can be partitioned into sets of functions which have either a local maximum, a local minimum, a saddle point which is four-arm in $B(R)$ or a saddle point which is $R$-upper/lower connected at the origin. Arguments which are very similar to those above show that each of these subsets is open, hence proving the statement of the lemma. (For saddle points which are four-arm in $B(R)$, we use the fact that $h(0)$ is not a critical level of $\left.h\right|_{\partial B(R)}$ which implies that the four level lines emanating from the origin intersect $\partial B(R)$ at different points.)

We next confirm that $\tilde{f}_{\ell} \in C_{\text {Reg }}^{2+\eta}(R)$ almost surely:

Lemma 4.3. If $f$ is a Gaussian field satisfying Assumptions 2.1 and 2.7, then for any $\ell \in \mathbb{R}$ and $R>0, \tilde{f}_{\ell} \in C_{R e g}^{2+\eta}(R)$ almost surely.

Proof. To simplify the presentation we assume that $\left(f(0), \nabla^{2} f(0)\right)$ is non-degenerate; the proof in the degenerate case is similar. Recall the representation of $\tilde{f}_{\ell}$ in Lemma 3.2. By the definitions of $\alpha, \beta$ and $\gamma, \tilde{f}_{\ell}$ is almost surely in $C_{\text {loc }}^{2+\eta}\left(\mathbb{R}^{2}\right)$, and has a critical point at the origin at level $\ell$. By evaluating the second order derivatives of $\alpha, \beta$ and $\gamma$, it follows that $\nabla^{2} \tilde{f}_{\ell}(0)=Z_{\ell}$. Since the density of $Z_{\ell}$ is identically zero on the region where its determinant is zero, $\operatorname{det} \nabla^{2} \tilde{f}_{\ell}(0) \neq 0$ almost surely, and so the critical point at the origin is non-degenerate.

Next we show that $\tilde{f}_{\ell}$ almost surely has no other critical points at level $\ell$. Let $T_{n}=\overline{B(n)} \backslash B\left(\frac{1}{n}\right)$ and consider $\left(\nabla \tilde{f}_{\ell}, \tilde{f}_{\ell}-\ell\right): T_{n} \rightarrow \mathbb{R}^{3}$. Bulinskaya's lemma ([1, Lemma 11.2.10]) states that this function almost surely has no zeroes in $T_{n}$ provided the univariate densities of $\left(\nabla \tilde{f}_{\ell}(t), \tilde{f}_{\ell}(t)\right)$ are bounded in a neighbourhood of $(0, \ell)$ uniformly over $t \in T_{n}$. Since $g$ and $Z_{\ell}$ are independent, the density of $\left(\nabla \tilde{f}_{\ell}(t), \tilde{f}_{\ell}(t)\right)$ is given by

$$
\begin{aligned}
p_{\nabla \tilde{f}_{\ell}(t), \tilde{f}_{\ell}(t)}(x) & =\int_{\mathbb{R}^{3}} p_{\nabla g(t), g(t)}(x-u) p_{\nabla\left(Z_{\ell} \cdot \beta(t)+\ell \alpha(t)\right), Z_{\ell} \cdot \beta(t)+\ell \alpha(t)}(u) d u \\
& \leq \sup _{x \in \mathbb{R}^{3}} p_{\nabla g(t), g(t)}(x) \int_{\mathbb{R}^{3}} p_{\nabla\left(Z_{\ell} \cdot \beta(t)+\ell \alpha(t)\right), Z_{\ell} \cdot \beta(t)+\ell \alpha(t)}(u) d u \\
& =\sup _{x \in \mathbb{R}^{3}} p_{\nabla g(t), g(t)}(x) .
\end{aligned}
$$

Therefore, to show that $p_{\nabla \tilde{f}_{\ell}(t), \tilde{f}_{\ell}(t)}$ is bounded, it is sufficient to show that the density of $(\nabla g(t), g(t))$ is bounded uniformly in $t$. Since these densities are Gaussian, this is equivalent to showing that the determinant of the covariance matrix of $(\nabla g(t), g(t))$ is bounded away from 0 on $T_{n}$. However this is the determinant of

$$
\operatorname{Cov}\left(\nabla f(t), f(t) \mid f(0), \nabla f(0), \nabla^{2} f(0)\right)
$$

which is non-degenerate for each $t \in T_{n}$ by Assumption 2.7. Since this determinant is continuous in $t$, it is bounded away from 0 on the compact set $T_{n}$. Taking the countable union of $T_{n}$ for $n \in \mathbb{N}$ then shows that $\tilde{f}_{\ell}$ almost surely has no critical points at level $\ell$ in $\mathbb{R}^{2} \backslash\{0\}$.

To verify that $\left.\tilde{f}_{\ell}\right|_{\partial B(R)}$ almost surely has no critical points at level $\ell$, we apply an identical argument to

$$
\left(\left(\begin{array}{c}
-\sin (\theta) \\
\cos (\theta)
\end{array}\right) \cdot \nabla \tilde{f}_{\ell}(y), \tilde{f}_{\ell}(y)\right)
$$

where $y=(R \cos (\theta), R \sin (\theta))$. This completes the proof that $\tilde{f}_{\ell} \in C_{\text {Reg }}^{2+\eta}(R)$ almost surely.

We are now ready to prove Theorem 2.11. Let $N_{s^{-}}^{(R)}\left[\ell_{1}, \ell_{2}\right]$ denote the number of $R$-lower connected saddle points of $f$ in $B(1)$ with height in $\left[\ell_{1}, \ell_{2}\right]$. If $N_{s^{-}}^{(R)}$ is replaced with $N_{s^{-}}$or $N_{s}$, we make a corresponding definition for lower connected saddle points 
or saddle points respectively. Recall that $s^{-}(R)$ is the subset of functions in $C_{\mathrm{Reg}}^{2+\eta}(R)$ with an $R$-lower connected saddle point at the origin. We also define $s^{-}$and $s^{+}$to be the subsets of $C_{\text {Reg }}^{2+\eta}$ with lower and upper connected saddle points at the origin respectively.

Proof of Theorem 2.11. Let $f$ be a field satisfying Assumptions 2.1 and 2.7. The first step is to show that $p_{s^{-}}^{*}$ is lower semi-continuous by expressing it as the pointwise supremum of a sequence of continuous functions. Let $\ell \in \mathbb{R}$ and $\epsilon>0$ and we fix $R>0$. We now claim that

$$
\frac{\mathbb{E}\left(N_{s^{-}}^{(R)}[\ell, \ell+\epsilon]\right)}{\mathbb{E}\left(N_{s}[\ell, \ell+\epsilon]\right)}=\mathbb{P}\left(\tilde{f}_{[\ell, \ell+\epsilon]} \in s^{-}(R)\right) .
$$

We first note that, by Lemma 4.2, the event $s^{-}(R)$ is contained in the Borel $\sigma$-algebra generated by the $C_{\mathrm{Reg}}^{2+\eta}(R)$ topology and that $\tilde{f}_{[\ell, \ell+\epsilon]}$ is measurable with respect to this $\sigma$-algebra. Furthermore, by an elementary argument (see, for example, [26, Lemma A.1]) this $\sigma$-algebra is generated by cylinder sets; those which depend on the value of the function at only finitely many points. Since the distribution of $\tilde{f}_{[\ell, \ell+\epsilon]}$ on cylinder sets is defined in (3.1) as an empirical measure, these two measures must coincide on the $\sigma$-algebra generated by this $\pi$-system. This verifies the claim.

By Lemma 3.1, $\tilde{f}_{[\ell, \ell+\epsilon]}$ converges in distribution to $\tilde{f}_{\ell}$ (in the $C_{\text {loc }}^{2+\eta}$ topology) as $\epsilon \rightarrow 0$, and since having a saddle point at the origin which is $R$-lower connected is a continuity event for $\tilde{f}_{\ell}$ (Lemmas 4.2 and 4.3 ), the portmanteau lemma implies that

$$
\mathbb{P}\left(\tilde{f}_{[\ell, \ell+\epsilon]} \in s^{-}(R)\right) \rightarrow \mathbb{P}\left(\tilde{f}_{\ell} \in s^{-}(R)\right)
$$

as $\epsilon \rightarrow 0$. By inspecting the form of $p_{Z_{\ell}}$ it is clear that $\tilde{f}_{\ell} \stackrel{d}{\rightarrow} \tilde{f}_{\ell_{0}}$ as $\ell \rightarrow \ell_{0}$ in the $C_{\text {loc }}^{2+\eta}$ topology. So by applying the portmanteau lemma again, we see that $\mathbb{P}\left(\tilde{f}_{\ell} \in s^{-}(R)\right)$ is continuous in $\ell$. Hence the function

$$
p_{s^{-}}^{(R)}(\ell):=p_{s}(\ell) \mathbb{P}\left(\tilde{f}_{\ell} \in s^{-}(R)\right),
$$

is continuous in $\ell$. Now note that

$$
\frac{1}{\epsilon} \mathbb{E}\left(N_{s^{-}}^{(R)}[\ell, \ell+\epsilon]\right)=\frac{\mathbb{E}\left(N_{s}[\ell, \ell+\epsilon]\right)}{\epsilon} \frac{\mathbb{E}\left(N_{s^{-}}^{(R)}[\ell, \ell+\epsilon]\right)}{\mathbb{E}\left(N_{s}[\ell, \ell+\epsilon]\right)} \rightarrow p_{s^{-}}^{(R)}(\ell)
$$

as $\epsilon \rightarrow 0$ (by Proposition 2.5). Hence $\mathbb{E}\left(N_{s^{-}}^{(R)}[-\infty, \ell]\right.$ ) is differentiable in $\ell$ with derivative $p_{s^{-}}^{(R)}(\ell)$. We now allow $R$ to vary; since $s^{-}(R)$ is non-decreasing in $R$ and $\cup_{R>0} s^{-}(R)=s^{-}$, taking the limit of (4.1) shows that

$$
p_{s^{-}}^{*}(\ell)=\lim _{R \rightarrow \infty} p_{s^{-}}^{(R)}(\ell)
$$

for each $\ell \in \mathbb{R}$. Hence $p_{s^{-}}^{*}$ is indeed a pointwise supremum of continuous functions, and so is lower semi-continuous.

We next prove that $p_{s^{-}}^{*}=p_{s^{-}}$almost everywhere. Let $a<b$, then since $\left|p_{s^{-}}^{*}-p_{s^{-}}\right|$is bounded, by dominated convergence

$$
\begin{aligned}
\int_{a}^{b} p_{s^{-}}(x)-p_{s^{-}}^{*}(x) d x & =\lim _{R \rightarrow \infty} \int_{a}^{b} p_{s^{-}}(x)-p_{s^{-}}^{(R)}(x) d x \\
& =\lim _{R \rightarrow \infty} \mathbb{E}\left(N_{s^{-}}[a, b]-N_{s^{-}}^{(R)}[a, b]\right)=0
\end{aligned}
$$

where in the last line we have used the definition of $p_{s^{-}}$, the fundamental theorem of calculus applied to $\mathbb{E}\left(N_{s^{-}}^{(R)}[-\infty, \ell]\right.$ ) (along with the differentiability proven above), and 
then dominated convergence once again. Since $a$ and $b$ are arbitrary, we conclude that $p_{s^{-}}^{*}=p_{s^{-}}$almost everywhere.

To finish the proof we show that (2.5), the condition that $\tilde{f}_{\ell}$ does not have an infinite four-arm saddle, implies the continuity of $p_{s^{*}}^{*}$. Observe that, by repeating the arguments above, we may define the lower semi-continuous function

$$
p_{s^{+}}^{*}(\ell):=p_{s}(\ell) \mathbb{P}\left(\tilde{f}_{\ell} \in s^{+}\right)
$$

which is a version of $p_{s^{+}}$. By Lemmas 4.1 and 4.3 , the saddle point of $\tilde{f}_{\ell}$ at the origin must be either upper connected, lower connected or an infinite four-arm saddle. Therefore

$$
1-\mathbb{P}\left(\tilde{f}_{\ell} \text { has an infinite four-arm saddle }\right)=\mathbb{P}\left(\tilde{f}_{\ell} \in s^{+} \cup s^{-}\right)=\frac{p_{s^{+}}^{*}(\ell)}{p_{s}(\ell)}+\frac{p_{s^{-}}^{*}(\ell)}{p_{s}(\ell)} .
$$

(Note that $p_{s}(\ell)>0$ by Lemma A.3.) Now suppose that (2.5) holds, that is, for all $\ell \in(a, b)$, $\tilde{f}_{\ell}$ almost surely does not have an infinite four-arm saddle point at the origin. By (4.2) we see that $p_{s^{+}}^{*}(\ell)=p_{s}(\ell)-p_{s^{-}}^{*}(\ell)$ for all $\ell \in(a, b)$. Since $p_{s^{+}}^{*}$ is lower semi-continuous (and $p_{s}$ is continuous), we deduce that $p_{s^{-}}^{*}$ is upper semi-continuous on $(a, b)$. Hence we have shown that $p_{s^{-}}^{*}$ is both upper and lower semi-continuous on $(a, b)$, which completes the result.

As mentioned previously, Theorem 2.10 follows from Theorem 2.11 once we verify condition (2.5). This is done in the next lemma:

Lemma 4.4. Let $f$ be a Gaussian field satisfying Assumptions 2.1 and 2.8-2.9. Then for every $\ell \geq 0$ and $r>0$,

$$
\mathbb{P}\left(\tilde{f}_{\ell} \in \operatorname{Arm}_{\ell}(r, R)\right) \rightarrow 0
$$

as $R \rightarrow \infty$. In particular, for all $\ell \in \mathbb{R}, \tilde{f}_{\ell}$ almost surely does not have an infinite four-arm saddle point at the origin.

Proof. We first note that if $\tilde{f}_{\ell}$ has an infinite four-arm saddle at the origin, then both $\left\{\tilde{f}_{\ell} \geq \ell\right\}$ and $\left\{\tilde{f}_{\ell} \leq \ell\right\}=\left\{-\tilde{f}_{\ell} \geq-\ell\right\}$ have unbounded components containing the origin. Then, since $\tilde{f}_{\ell}$ and $-\tilde{f}_{-\ell}$ have the same distribution by Lemma 3.2 , the second claim of this lemma follows from the first.

Since the event $\operatorname{Arm}_{\ell}(r, R)$ is weakly increasing in $r$, it is sufficient to prove (4.3) for a sequence $r_{R} \rightarrow \infty$ as $R \rightarrow \infty$. This allows us to make use of the fact that, far from the origin, the distribution of $\tilde{f}_{\ell}$ is close to that of $f$.

By Assumption 2.8 and Lemma A.2 we know that $\left(f(0), \nabla^{2} f(0)\right)$ is non-degenerate. Recall the representation for $\tilde{f}_{\ell}$ in Lemma 3.2

$$
\tilde{f}_{\ell}=g+\ell \alpha+Z_{\ell} \cdot \beta
$$

and recall also the explicit expressions for $\alpha, \beta$ and the covariance of $g$ derived after this lemma. Since this covariance is expressed as the difference of two positive definite functions, if we let $f_{1}$ be a centred Gaussian field with covariance

$$
K_{1}(s, t)=\operatorname{Cov}(f(s), v) \Sigma^{-1} \operatorname{Cov}(f(t), v)^{\prime},
$$

then we can decompose $f=g+f_{1}$, where $f_{1}$ and $g$ are independent. Since $K_{1}$ can be expressed as a linear combination of $\partial^{k_{1}} K(s) \partial^{k_{2}} K(t)$, for $\left|k_{1}\right|,\left|k_{2}\right| \leq 2$, by Assumption 2.8 there exists $c_{1}, \nu>0$ such that, for all $r>1$,

$$
\sup _{s, t \notin B(r)} \sup _{|k| \leq 2}\left|\partial^{k} K_{1}(s, t)\right| \leq c_{1} r^{-2(1+\nu)} .
$$


Moreover, since $\alpha, \beta$ can be expressed as a linear combination of $\partial^{k} K(t)$, for $|k| \leq 2$, by Assumption 2.8 there exists $c_{2}, \nu>0$ such that

$$
\sup _{|t|>r}|\alpha(t)| \leq c_{2} r^{-(1+\nu)} \quad \text { and } \quad \sup _{|t|>r}\|\beta(t)\|_{\infty} \leq c_{2} r^{-(1+\nu)} .
$$

Next, we fix $\ell \geq 0$ and apply a Cameron-Martin argument to the unconditional field $f$. Specifically, by [25, Corollary 3.7] (valid by the condition on the spectral density in Assumption 2.8, and since $\operatorname{Arm}_{\ell}(r, R)$ is an increasing event with respect to the field) there exists $c_{3}, r_{0}>0$ such that for all $r>r_{0}$ the following holds: if $F: \mathbb{R}^{2} \rightarrow \mathbb{R}$ is a continuous random field coupled with $f$ such that

$$
\mathbb{P}\left(\|f-F\|_{\infty, A(r, R)} \geq \epsilon\right) \leq \delta
$$

where $\|\cdot\|_{\infty, A(r, R)}$ denotes the supremum norm on $A(r, R)$ the centred annulus of inner radius $r$ and outer radius $R$, then

$$
\mathbb{P}\left(F \in \operatorname{Arm}_{\ell}(r, R)\right) \leq \mathbb{P}\left(f \in \operatorname{Arm}_{\ell}(r, R)\right)+\delta+c_{3} R \epsilon .
$$

We will apply this bound to $F=\tilde{f}_{\ell}$. Note that, by the union bound,

$$
\begin{aligned}
& \mathbb{P}\left(\left\|f-\tilde{f}_{\ell}\right\|_{\infty, A(r, R)} \geq \epsilon\right) \\
& \leq \mathbb{P}\left(\left\|\ell \alpha+Z_{\ell} \cdot \beta\right\|_{\infty, A(r, R)} \geq \epsilon / 2\right)+\mathbb{P}\left(\left\|f_{1}\right\|_{\infty, A(r, R)} \geq \epsilon / 2\right) \\
& \leq \mathbb{1}\left\{\ell\|\alpha\|_{\infty, A(r, R)} \geq \epsilon / 8\right\}+\sum_{i \in\{11,12,22\}} \mathbb{P}\left(\mid Z_{\ell, i}\left\|\beta_{i}\right\|_{\infty, A(r, R)} \geq \epsilon / 8\right) \\
&+\mathbb{P}\left(\left\|f_{1}\right\|_{\infty, A(r, R)} \geq \epsilon / 2\right)
\end{aligned}
$$

where $Z_{\ell, i}$ denotes the elements of the random vector $Z_{\ell}$. We now show that, for a suitable choice of $r=r_{R} \rightarrow \infty$ and $\epsilon=\epsilon_{R} \rightarrow 0$, the three terms in (4.7) all decay to zero as $R \rightarrow \infty$.

By (4.5), and since $Z_{\ell, i}$ is almost surely finite, the first two terms in (4.7) converge to zero as long as $\epsilon r^{1+\nu} \rightarrow \infty$. If we assume this convergence is sufficiently fast (to be specified below) then it is a standard estimate for the norm of a Gaussian field that the third term of (4.7) also converges to zero. This argument is essentially the same as [25, Lemma 3.12], but our setting is slightly different so we give a complete proof.

Let $B_{x}(1)$ denote the ball of radius 1 centred at $x$. Covering $A(r, R)$ with $O\left(R^{2}\right)$ unit balls, and by the union bound,

$$
\mathbb{P}\left(\left\|f_{1}\right\|_{\infty, A(r, R)} \geq \epsilon / 2\right) \leq c_{3} R^{2} \sup _{x \in A(r, R)} \mathbb{P}\left(\left\|f_{1}\right\|_{\left.\infty, B_{x}(1)\right)} \geq \epsilon / 2\right) .
$$

By the Borell-TIS inequality ([1, Theorem 2.1.1]), for all $u>0$,

$$
\mathbb{P}\left(\left\|f_{1}\right\|_{\left.\infty, B_{x}(1)\right)} \geq m_{x}+u\right) \leq 2 e^{-u^{2} /\left(2 \sigma_{x}^{2}\right)},
$$

where

$$
m_{x}=\mathbb{E}\left[\left\|f_{1}\right\|_{\infty, B_{x}(1)}\right] \quad \text { and } \quad \sigma_{x}^{2}=\sup _{y \in B_{x}(1)} K_{1}(y, y) .
$$

By Kolmogorov's theorem [26, Appendix A.9], there is a $c_{4}>0$ such that

$$
m_{x}<c_{4} \sup _{s, t \in B_{x}(1)} \sup _{\left|\alpha_{1}\right|,\left|\alpha_{2}\right| \leq 1}\left(\partial^{\alpha_{1}, \alpha_{2}} K_{1}(s, t)\right)^{1 / 2} .
$$

Therefore, by (4.4),

$$
\sup _{x \in A(r, R)} m_{x}<c_{5} r^{-1-\nu} \text { and } \sup _{x \in A(r, R)} \sigma_{x}^{2}<c_{5} r^{-2-2 \nu} .
$$


Taking $u=\epsilon / 4$ and assuming that $\epsilon / 4>c_{5} r^{-1-\nu}$ we have

$$
\mathbb{P}\left(\left\|f_{1}\right\|_{\infty, A(r, R)} \geq \epsilon / 2\right) \leq 2 c_{6} R^{2} \exp \left(-c_{7} \epsilon^{2} r^{2+2 \nu}\right)
$$

To finish, we take

$$
r=\frac{R}{\log (R)} \quad \text { and } \quad \epsilon=\frac{1}{R \log (R)}
$$

and observe that for this choice the right hand side of the estimate above converges to 0 as $R \rightarrow \infty$. Combining all of these estimates together we have that the right hand side of (4.7) tends to zero as $R \rightarrow \infty$.

Substituting into (4.6), and noting that $r / R \rightarrow 0$ and $R \epsilon \rightarrow 0$ as $R \rightarrow \infty$, proves that $\mathbb{P}\left(\tilde{f}_{\ell} \in \operatorname{Arm}_{\ell}(r, R)\right)$ can be made arbitrarily small, which completes the proof of the lemma.

Proof of Theorem 2.10. This is immediate from Theorem 2.11 and Lemma 4.4.

To end the section we prove the remaining results stated in Section 2.1, namely Corollary 2.12 and Proposition 2.14.

Proof of Corollary 2.12. By [6, Lemmas 2.4 and 4.5], $\mathbb{E}\left(N_{4-\operatorname{arm}}(R)\right)=O(R)$ as $R \rightarrow \infty$, so it suffices to prove the other bound here. Recall that $A(R-r, R)$ denotes the annulus of inner radius $R-r$ and outer radius $R$. We first note that for any $1<r<R$

$$
N_{4-\operatorname{arm}}\left(R,\left[a_{R}, b_{R}\right]\right) \leq N_{\mathrm{c}}\left(A(R-r, R),\left[a_{R}, b_{R}\right]\right)+N_{4-\operatorname{arm}, r}\left(B(R-r),\left[a_{R}, b_{R}\right]\right)
$$

where, by a slight abuse of notation, $N_{\mathrm{c}}\left(A(R-r, R),\left[a_{R}, b_{R}\right]\right)$ denotes the number of critical points in $A(R-r, R)$ which have level in $\left[a_{R}, b_{R}\right]$, and $N_{4-\operatorname{arm}, r}\left(B(R-r),\left[a_{R}, b_{R}\right]\right)$ denotes the number of saddle points $t \in B(R-r)$ which are four-arm in $B(t, r)$ and have level in $\left[a_{R}, b_{R}\right]$. Using the Kac-Rice theorem ([1, Corollary 11.2.2]) and the independence of $\left(f(0), \nabla^{2} f(0)\right)$ and $\nabla f(0)$

$$
\begin{aligned}
\mathbb{E}\left(N_{c}(A\right. & \left.\left.(R-r, R),\left[a_{R}, b_{R}\right]\right)\right) \\
& =\int_{A(R-r, R)} \mathbb{E}\left(\left|\operatorname{det}\left(\nabla^{2} f(0)\right)\right| \mathbb{1}_{f(0) \in\left[a_{R}, b_{R}\right]} \mid \nabla f(0)=0\right) p_{\nabla f(0)}(0) d t \\
& =c_{1}\left(R^{2}-(R-r)^{2}\right) \int_{a_{R}}^{b_{R}} \mathbb{E}\left(\mid \operatorname{det}\left(\nabla^{2} f(0)\right) \| f(0)=x\right) p_{f(0)}(x) d x \\
& \leq c_{2} R r \cdot\left(b_{R}-a_{R}\right)
\end{aligned}
$$

for some $c_{1}, c_{2}>0$ independent of $R$. By stationarity of $f$

$$
\begin{aligned}
\mathbb{E}\left(N_{4-\operatorname{arm}, r}\left(B(R-r),\left[a_{R}, b_{R}\right]\right)\right) & \leq R^{2} \mathbb{E}\left(N_{4-\operatorname{arm}, r}\left(B(1),\left[a_{R}, b_{R}\right]\right)\right) \\
& =\pi R^{2} \int_{a_{R}}^{b_{R}} p_{s}(x)-p_{s^{-}}^{(r)}(x)-p_{s^{+}}^{(r)}(x) d x
\end{aligned}
$$

where $p_{s^{-}}^{(r)}$ and $p_{s^{+}}^{(r)}$ are the continuous functions defined as in the proof of Theorem 2.11. In this proof it is shown that as $r \rightarrow \infty, p_{s^{-}}^{(r)}+p_{s^{+}}^{(r)}$ converges pointwise monotonically to $p_{s^{-}}^{*}+p_{s^{+}}^{*}=p_{s}$ which is continuous. Therefore by Dini's theorem this convergence is uniform on $[a, b]$ and so for any $\epsilon>0$ taking $r$ sufficiently large relative to $\epsilon$ ensures that the right hand side of (4.9) is bounded above by $\epsilon R^{2}\left(b_{R}-a_{R}\right)$. If we choose $r$ depending on $R$ such that $r \rightarrow \infty$ but $r / R \rightarrow 0$ as $R \rightarrow \infty$, then combining (4.8) and (4.9) proves the corollary. 
Proof of Proposition 2.14. By Theorem 2.6 and the identities in Proposition 2.5,

$$
c_{L S}(\ell)=c_{E S}(\ell)+c_{E S}(-\ell) .
$$

Let us also consider the function

$$
h(\ell)=c_{E S}(\ell)-c_{E S}(-\ell)
$$

In [6, Corollary 1.12], this is interpreted as the asymptotic mean Euler characteristic of the excursion set at level $\ell$, and hence shown via explicit calculation to be equal to the $C^{1}$ function

$$
h(\ell)=\sqrt{\operatorname{det} \nabla^{2} K(0)} \frac{\ell}{(2 \pi)^{3 / 2}} e^{-\ell^{2} / 2} .
$$

If $c_{L S}(0)=0$, then it follows from (4.10) that $c_{E S}(0)=0$. Similarly, if $c_{L S}$ is differentiable at 0 , then by (4.10) and the differentiability of $h, c_{E S}$ is also differentiable at 0.

It remains to show that if $c_{E S}$ is differentiable at 0 then $c_{E S}(0) \neq 0$. Suppose for the sake of contradiction that $c_{E S}(0)=0$. Then by the non-negativity of $c_{E S}$, we have $c_{E S}^{\prime}(0)=0$. Hence, by $(4.11), h^{\prime}(0)=0$. Since $h$ has critical points only at $\ell= \pm 1$, we have derived the necessary contradiction.

Remark 4.5. Assuming differentiability of $c_{E S}$ or $c_{L S}$ at $\ell$, the above argument actually shows that $c_{L S}(\ell)>0$ for all $\ell \neq \pm 1$ (although it apparently says nothing about the positivity of $c_{E S}(\ell)$ for $\left.\ell \neq 0\right)$.

\section{Monotonicity results}

In this section we prove the monotonicity results stated in Section 2.2. The main intermediate step is to show that the finite-dimensional projections of $\tilde{f}_{\ell}-\ell$ are stochastically decreasing in $\ell$, which we do in the next subsection.

\subsection{Stochastic monotonicity}

Our analysis differs depending on whether we deal with the RPW or a general isotropic field satisfying Assumption 2.15, the RPW case being somewhat simpler.

\subsubsection{Stochastic monotonicity for the RPW}

Let $f$ be the RPW. The first step is to show, via explicit calculation, that $\tilde{f}_{\ell}-\ell$ is stochastically decreasing in $\ell$ at every point.

By Proposition 3.4, $\tilde{f}_{\ell}$ has the distribution

$$
\tilde{f}_{\ell}(t)=g(t)+\ell \cdot\left[J_{0}(|t|)+2 \cos (2(\theta-\arg t)) J_{2}(|t|)\right]+\lambda \cdot 4 \cos (2(\theta-\arg t)) J_{2}(|t|)
$$

for the random vector $(\theta, \lambda)$ defined in that proposition. To simplify notation, we define

$$
\begin{aligned}
a & :=a(t, \theta)=1-J_{0}(|t|)-2 \cos (2(\theta-\arg t)) J_{2}(|t|) \\
b & :=b(t, \theta)=4 \cos (2(\theta-\arg t)) J_{2}(|t|) .
\end{aligned}
$$

The key fact leading to stochastic monotonicity is that, by Lemma 5.1 below, $a(t, \theta) \geq 0$ for all $t$ and $\theta$. This is equivalent to the statement that for all $t \in \mathbb{R}^{2}$

$$
\alpha(t)=\mathbb{E}\left(f(t) \mid f(0)=1, f_{11}(0)=f_{12}(0)=0\right) \leq 1 .
$$

For general isotropic fields, we show in Lemma 5.6 that Assumption 2.15 implies $\alpha(t) \leq 1$ (recall that $\alpha$ has a slightly different definition in the general case, see Lemma 3.2). 
Lemma 5.1. For all $t \in \mathbb{R}^{2}$ and $\theta \in \mathbb{R}$,

$$
a=a(t, \theta)=1-J_{0}(|t|)-2 \cos (2(\theta-\arg t)) J_{2}(|t|) \geq 0 .
$$

Proof. It is sufficient to prove that, for $s \geq 0$,

$$
1-J_{0}(s)-2 J_{2}(s) \geq 0 \text { and } 1-J_{0}(s)+2 J_{2}(s) \geq 0 .
$$

By the identity $2 J_{1}(s) / s=J_{0}(s)+J_{2}(s)$ and an explicit uniform bound on $\sqrt{s}\left|J_{n}(s)\right|$ given in [27, Theorem 2.1], the first inequality holds for all $s>4$. Hence, since $1-J_{0}(0)-$ $2 J_{2}(0)=0$ and $\frac{d}{d s}\left(1-J_{0}(s)-2 J_{2}(s)\right)=J_{3}(s)$ (which is non-negative for $s \in[0,4]$ ), the first inequality holds for all $s \geq 0$.

The same bound from [27] shows that the second inequality holds for $s \geq 11$. Since $\left|J_{0}\right| \leq 1$ everywhere and $J_{2}(s) \geq 0$ for $s \in[0,5] \cup[9,11]$, the inequality also holds on these intervals. We verify the second inequality on the remaining compact set $[5,9]$ by inspection. More precisely, since

$$
\left|\frac{d}{d s}\left(1-J_{0}(s)+2 J_{2}(s)\right)\right|=\left|2 J_{1}(s)-J_{3}(s)\right| \leq 2\left|J_{1}(s)\right|+\left|J_{3}(s)\right|<2,
$$

it suffices to check that $1-J_{0}(s)+2 J_{2}(s)>0.08$ for all $s \in\{5+4 i / 100: i=0,1, \ldots, 100\}$.

Remark 5.2. We prove the above lemma by somewhat explicit computations. We believe that there might be a more conceptual proof of this statement.

We shall actually show the slightly stronger statement that $\tilde{f}_{\ell}-\ell$ is pointwise stochastically decreasing conditional on all values of $(g, \theta)$ :

Lemma 5.3. Let $f$ be the $R P W$. For $t \in \mathbb{R}^{2}$ and $c \in \mathbb{R}$

$$
\mathbb{P}\left(\tilde{f}_{\ell}(t)-\ell \leq c \mid g, \theta\right)
$$

is non-decreasing in $\ell \in \mathbb{R}$.

Proof. Given the representation in (5.1), we have

$$
\mathbb{P}\left(\tilde{f}_{\ell}(t)-\ell \leq c \mid g, \theta\right)= \begin{cases}\mathbb{P}(\lambda \leq(c-g(t)+a \ell) / b) & \text { if } b(t, \theta)>0, \\ \mathbb{P}(\lambda \geq(c-g(t)+a \ell) / b) & \text { if } b(t, \theta)<0 \\ \mathbb{1}_{a \ell+c-g(t) \geq 0} & \text { if } b(t, \theta)=0 .\end{cases}
$$

It remains to show that each of the expressions on the right-hand side of (5.2) are non-decreasing in $\ell$ for all values of $g, \theta$ and $c$. Recall that $a=a(t, \theta) \geq 0$ by Lemma 5.1. Hence $\mathbb{1}_{a \ell+c-g(t) \geq 0}$ is clearly non-decreasing in $\ell$. Moreover, after integrating (3.4), we see that for a differentiable function $h: \mathbb{R} \rightarrow \mathbb{R}$

$$
\frac{d}{d \ell} \mathbb{P}(\lambda \leq h(\ell))=p_{\lambda}(h)\left(h^{\prime}(\ell)+\frac{h(\ell)}{2 h(\ell)+\ell}\right) \leq p_{\lambda}(h)\left(h^{\prime}(\ell)+1\right)
$$

where the last inequality follows from the fact that $p_{\lambda}(h)$ is zero unless $h>0 \vee(-\ell)$. Now let $h(\ell)=(c-g(t)+a \ell) / b$, and first suppose $b>0$. Then $h^{\prime}(\ell)=a / b>0, h /(2 h+\ell) \geq 0$ on the region $h>0 \vee(-\ell)$ and $p_{\lambda}(h) \geq 0$ (as a probability density) so (5.3) shows that the left hand side of (5.2) is non-decreasing whenever $b>0$. Finally we suppose $b<0$ and note that

$$
h^{\prime}(\ell)+1=\frac{a(t, \theta)+b(t, \theta)}{b(t, \theta)}=\frac{1-J_{0}(|t|)+2 \cos (2(\theta-\arg t)) J_{2}(|t|)}{b(t, \theta)}=\frac{a(t, \theta+\pi / 2)}{b(t, \theta)} \leq 0 .
$$

So once again, (5.3) shows the left hand side of (5.2) is non-decreasing whenever $b<0$, completing the proof of the lemma. 
We now extend this result to finite-dimensional projections of $\tilde{f}_{\ell}-\ell$. Recall that a random vector $X=\left(X_{1}, \ldots, X_{n}\right)$ is said to stochastically dominate a random vector $Y=$ $\left(Y_{1}, \ldots, Y_{n}\right)$, written $X \succ Y$, if $\mathbb{E}(g(X)) \geq \mathbb{E}(g(Y))$ for any coordinate-wise increasing $g: \mathbb{R}^{n} \rightarrow \mathbb{R}$. Clearly, if $X \succ Y$ then $X_{i} \succ Y_{i}$ for each $i=1, \ldots, n$. The converse is not true in general, but a useful sufficient condition can be formulated using the notion of copulas.

Let $X=\left(X_{1}, \ldots, X_{n}\right)$, where $X_{i}$ has cumulative density function $F_{i}$ and induced probability measure $\mathbb{P}_{i}$. Then Sklar's theorem states that there exists a (unique on $\left.\Pi_{i=1}^{n} \operatorname{Range}\left(\mathbb{P}_{i}\right)\right)$ function $\operatorname{Cop}_{X}:[0,1]^{n} \rightarrow[0,1]$, known as the copula of $X$, such that

$$
\mathbb{P}\left(X \in A_{1} \times \cdots \times A_{n}\right)=\operatorname{Cop}_{X}\left(\mathbb{P}_{1}\left(A_{1}\right), \ldots, \mathbb{P}_{n}\left(A_{n}\right)\right)
$$

for all $A_{1}, \ldots, A_{n} \in \mathcal{B}(\mathbb{R})$. The copula is equivalently specified by

$$
\operatorname{Cop}_{X}\left(u_{1}, \ldots, u_{n}\right)=\mathbb{P}\left(F_{1}\left(X_{1}\right) \leq u_{1}, \ldots, F_{n}\left(X_{n}\right) \leq u_{n}\right) \text {, }
$$

i.e. $\operatorname{Cop}_{X}$ is the joint cumulative density function of the collection of uniform-[0,1] random variables $F_{1}\left(X_{1}\right), \ldots, F_{n}\left(X_{n}\right)$.

Theorem 5.4 ([32, Theorem 2]). Let $X=\left(X_{1}, \ldots, X_{n}\right)$ and $Y=\left(Y_{1}, \ldots, Y_{n}\right)$ be random vectors with induced marginal probability measures $\mathbb{P}_{1}, \ldots, \mathbb{P}_{n}$ and $\mathbb{Q}_{1}, \ldots, \mathbb{Q}_{n}$ respectively. If $\operatorname{Cop}_{X}=\operatorname{Cop}_{Y}, \Pi_{i=1}^{n} \operatorname{Range}\left(\mathbb{P}_{i}\right)=\Pi_{i=1}^{n} \operatorname{Range}\left(\mathbb{Q}_{i}\right)$, and $X_{i} \succ Y_{i}$ for each $i$, then $X \succ Y$.

Using this theorem, we extend Lemma 5.3 to show the stochastic monotonicity of the finite-dimensional projections $\tilde{f}_{\ell}-\ell$, conditional on any $g, \theta$.

Lemma 5.5. Let $f$ be the RPW. For $\ell_{1}<\ell_{2}$ and $t_{1}, \ldots, t_{n} \in \mathbb{R}^{2}$,

$$
\left(\tilde{f}_{\ell_{1}}\left(t_{1}\right)-\ell_{1}, \ldots, \tilde{f}_{\ell_{1}}\left(t_{n}\right)-\ell_{1} \mid g, \theta\right) \succ\left(\tilde{f}_{\ell_{2}}\left(t_{1}\right)-\ell_{2}, \ldots, \tilde{f}_{\ell_{2}}\left(t_{n}\right)-\ell_{2} \mid g, \theta\right) .
$$

Proof. By Theorem 5.4 it is sufficient to show that the random vectors in (5.5) have the same copula (these copulas are uniquely defined on the same domain, and the stochastic domination of the marginals follows from Lemma 5.3).

Fix $\ell \in \mathbb{R}$ and $t_{1}, \ldots, t_{n} \in \mathbb{R}^{2}$, and consider the copula

$$
\operatorname{Cop}_{Z}:=\operatorname{Cop}_{\tilde{f}_{\ell}\left(t_{1}\right)-\ell, \ldots, \tilde{f}_{\ell}\left(t_{n}\right)-\ell \mid g, \theta} \text {. }
$$

By the definition of $a$ and $b$, we can express

$$
\tilde{f}_{\ell}(t)-\ell=g(t)-\ell a(t, \theta)+\lambda_{\ell} b(t, \theta)
$$

for deterministic functions $a, b$. Since $g\left(t_{i}\right), \ell a\left(t_{i}, \theta\right)$ and $b\left(t_{i}, \theta\right)$ are constants under the conditioning on $(g, \theta)$, and since copulas are invariant under strictly increasing transformations,

$$
\operatorname{Cop}_{Z}=\operatorname{Cop}_{\lambda_{\ell} \cdot \operatorname{sign}\left(b\left(t_{1}, \theta\right)\right), \ldots, \lambda_{\ell} \cdot \operatorname{sign}\left(b\left(t_{n}, \theta\right)\right) \mid g, \theta}=\operatorname{Cop}_{\lambda_{\ell} \cdot \operatorname{sign}\left(b\left(t_{1}, \theta\right)\right), \ldots, \lambda_{\ell} \cdot \operatorname{sign}\left(b\left(t_{n}, \theta\right)\right) \mid \theta^{\prime}}
$$

where the last equality holds since $g$ is independent of $\lambda=\lambda_{\ell}$ and $\theta$. Notice that the random vector $\left(\lambda_{\ell} \cdot \operatorname{sign}\left(b\left(t_{1}, \theta\right)\right), \ldots, \lambda_{\ell} \cdot \operatorname{sign}\left(b\left(t_{n}, \theta\right)\right) \mid \theta\right)$ consists of $\lambda_{\ell}$ multiplied by a constant vector (with elements taking values $1,-1$ or 0 ). Hence by considering the alternative characterisation of a copula in (5.4), it is clear that $\operatorname{Cop}_{Z}$ does not depend on the distribution of $\lambda_{\ell}$, and so is independent of $\ell$. 


\subsubsection{Stochastic monotonicity in the general isotropic case}

The copula argument in the RPW case relies crucially on the degeneracy of RPW, which implies that after conditioning on $\theta$ and $g$, the field depends only on the single random variable $\lambda$. For general isotropic fields, $\tilde{f}_{\ell}$ is defined in terms of two eigenvalues, so this argument fails. Instead we use a different method that works with the finite-dimensional projections directly.

Let $f$ satisfy Assumptions 2.1, 2.7 and 2.15. Recall from Proposition 3.3 that

$$
\tilde{f}_{\ell}(t)=g(t)+\ell \alpha(t)+\lambda_{1} b_{1}(t, \theta)+\lambda_{2} b_{2}(t, \theta)
$$

for $\alpha, b_{1}, b_{2}$ as stated in the proposition (recall that $b_{1}$ and $b_{2}$ are defined in terms of $\beta$ ). The role of Assumption 2.15 is to ensure the following inequalities hold for $\alpha, b_{1}, b_{2}$ :

Lemma 5.6. Let $f$ satisfy Assumptions $2.1,2.7$ and 2.15. For all $t \in \mathbb{R}^{2}$ and $\theta \in[0,2 \pi)$,

$$
b_{1}(t, \theta) \geq 0, \quad b_{2}(t, \theta) \geq 0 \quad \text { and } \quad \alpha(t) \leq 1 .
$$

Proof. From the definition of $b_{1}(t, \theta)$ and $\beta$, it is immediate that $b_{1}(t, 0)$ is the quantity given in Assumption 2.15 to be non-negative for all values of $t$. Since $f$ is isotropic, $b_{1}$ is non-negative for all values of $\theta$. (By the identity $\cos (\theta)=\sin (\theta+\pi / 2$ ), this also means that $b_{2}$ is non-negative.) Similarly, $\alpha$ is the function given in Assumption 2.15 to be bounded above by 1 .

Lemma 5.7. Let $f$ satisfy Assumptions $2.1,2.7$ and 2.15. For any $t_{1}, \ldots, t_{n} \in \mathbb{R}^{2}$ and $u_{1}, \ldots, u_{n} \in \mathbb{R}$,

$$
\mathbb{P}\left(\tilde{f}_{\ell}\left(t_{i}\right)-\ell \leq u_{i} \forall i=1, \ldots, n \mid g, \theta\right)
$$

is non-decreasing in $\ell \in \mathbb{R}$.

Proof. Since the $u_{i}$ are arbitrary, we may assume $g\left(t_{i}\right)=0$ for all $i$. We define the region

$$
A_{\ell}=\left\{(x, y) \in \mathbb{R}^{2}: x b_{1}\left(t_{i}, \theta\right)+y b_{2}\left(t_{i}, \theta\right) \leq u_{i}+\left(1-\alpha\left(t_{i}\right)\right) \ell, \quad \forall i=1, \ldots, n\right\}
$$

so that $\tilde{f}_{\ell}\left(t_{i}\right)-\ell \leq u_{i}$ for all $i$ if and only if $\left(\lambda_{1}, \lambda_{2}\right) \in A_{\ell}$. It is enough to prove that the probability of the latter event is non-decreasing in $\ell$ because $\left(\lambda_{1}, \lambda_{2}\right)$ is independent of $(g, \theta)$. Given the density of $\left(\lambda_{1}, \lambda_{2}\right)$ in Proposition 3.3,

$$
\begin{aligned}
\frac{d}{d \ell} \mathbb{P}\left(\left(\lambda_{1}, \lambda_{2}\right) \in A_{\ell}\right) & =\frac{d}{d \ell} \frac{\int_{A_{\ell}} q_{\ell}(x, y) d x d y}{\int_{\mathbb{R}^{2}} q_{\ell}(x, y) d x d y} \\
& =\frac{\int_{\mathbb{R}^{2}} q_{\ell} d x d y \cdot \frac{d}{d \ell} \int_{A_{\ell}} q_{\ell} d x d y-\int_{A_{\ell}} q_{\ell} d x d y \cdot \frac{d}{d \ell} \int_{\mathbb{R}^{2}} q_{\ell} d x d y}{\left(\int_{\mathbb{R}^{2}} q_{\ell} d x d y\right)^{2}} .
\end{aligned}
$$

Since $b_{1}, b_{2} \geq 0$ and $\alpha \leq 1$ by Lemma 5.6, $A_{\ell}$ is non-decreasing in $\ell$, and so for this derivative to be non-negative it is sufficient that

$$
\frac{\int_{A_{\ell}} \frac{d}{d \ell} q_{\ell}(x, y) d x d y}{\int_{A_{\ell}} q_{\ell}(x, y) d x d y} \geq \frac{\int_{\mathbb{R}^{2}} \frac{d}{d \ell} q_{\ell}(x, y) d x d y}{\int_{\mathbb{R}^{2}} q_{\ell}(x, y) d x d y} .
$$

By direct evaluation

$$
\frac{d}{d \ell} q_{\ell}(x, y)=\frac{\mu(1+\tau)}{\sigma^{2}}(x+y-2 \mu \ell) q_{\ell}(x, y),
$$

where $\mu, \tau$ and $\sigma^{2}$ are defined in (3.3). Since $\mu<0,(5.6)$ is equivalent to

$$
\mathbb{E}\left(\lambda_{1}+\lambda_{2} \mid\left(\lambda_{1}, \lambda_{2}\right) \in A_{\ell}\right) \leq \mathbb{E}\left(\lambda_{1}+\lambda_{2}\right) .
$$


To complete the proof of the lemma, we show that this inequality holds for any possible region $A_{\ell}$. Since the shape of $A_{\ell}$ might be quite complicated (see Figure 5 for a typical example), we divide the analysis into three cases and in each case show that conditioning on $\left(\lambda_{1}, \lambda_{2}\right)$ being contained in some simple region can only increase the expectation of $\lambda_{1}+\lambda_{2}$ relative to conditioning on $\left(\lambda_{1}, \lambda_{2}\right) \in A_{\ell}$.

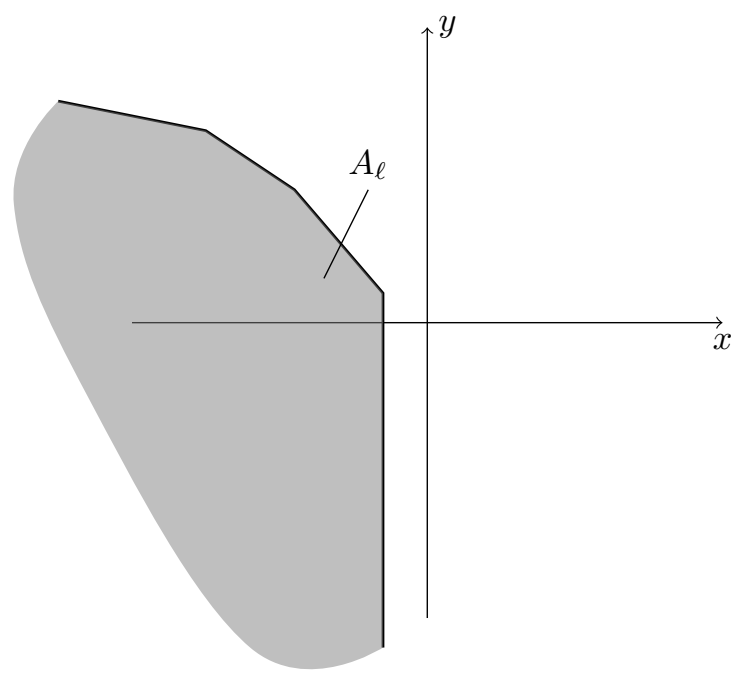

Figure 5: A typical example of the region $A_{\ell}$.

(Case 1). Suppose $b_{1}\left(t_{i}, \theta\right) / b_{2}\left(t_{i}, \theta\right)=1$ for all $i$, so that the boundary of $A_{\ell}$, denoted $\partial A_{\ell}$, is a line of the form $x+y=c$ for some $c \in \mathbb{R}$. (Note that $A_{\ell}$ is a subset of the entire plane not just the upper-left quadrant which is the support of $\left(\lambda_{1}, \lambda_{2}\right)$.) Then trivially

$$
\mathbb{E}\left(\lambda_{1}+\lambda_{2} \mid\left(\lambda_{1}, \lambda_{2}\right) \in A_{\ell}\right)=\mathbb{E}\left(\lambda_{1}+\lambda_{2} \mid \lambda_{1}+\lambda_{2} \leq c\right) \leq \mathbb{E}\left(\lambda_{1}+\lambda_{2}\right)
$$

and so (5.7) is verified in this case.

(Case 2). Now suppose that $b_{1}\left(t_{i}, \theta\right) / b_{2}\left(t_{i}, \theta\right) \geq 1$ for all $i$ and that this inequality is strict for some $i$ (allowing for the degenerate case that $b_{2}\left(t_{i}, \theta\right)=0$ ). Let $c^{*}=$ $\mathbb{E}\left(\lambda_{1}+\lambda_{2} \mid\left(\lambda_{1}, \lambda_{2}\right) \in A_{\ell}\right)$ and then we note that the line $x+y=c^{*}$ must intersect $\partial A_{\ell}$ at precisely one point (since the distribution of $\left(\lambda_{1}, \lambda_{2}\right)$ is continuous) which we denote by $\left(d_{1}, d_{2}\right)$. We now consider the region $\left\{x \leq d_{1}\right\}$, conditioning on $\left(\lambda_{1}, \lambda_{2}\right)$ lying in this region weakly increases the probability that $\lambda_{1}+\lambda_{2}=c$ for $c \geq c^{*}$ and weakly decreases this probability for $c<c^{*}$ (see Figure 6). Therefore

$$
\mathbb{E}\left(\lambda_{1}+\lambda_{2} \mid\left(\lambda_{1}, \lambda_{2}\right) \in A_{\ell}\right) \leq \mathbb{E}\left(\lambda_{1}+\lambda_{2} \mid \lambda_{1} \leq d_{1}\right) .
$$

If $b_{1}\left(t_{i}, \theta\right) / b_{2}\left(t_{i}, \theta\right) \leq 1$ for all $i$ and this inequality is strict for some $i$, then an entirely analogous argument shows that for some $d_{2}$

$$
\mathbb{E}\left(\lambda_{1}+\lambda_{2} \mid\left(\lambda_{1}, \lambda_{2}\right) \in A_{\ell}\right) \leq \mathbb{E}\left(\lambda_{1}+\lambda_{2} \mid \lambda_{2} \leq d_{2}\right) .
$$

(Case 3). Suppose that for some $i$ and $j, b_{1}\left(t_{i}, \theta\right) / b_{2}\left(t_{i}, \theta\right)<1<b_{1}\left(t_{j}, \theta\right) / b_{2}\left(t_{j}, \theta\right)$. Defining $c^{*}$ as before we note that the line $x+y=c^{*}$ must intersect $A_{\ell}$ (by definition of $\left.c^{*}\right)$ and so must intersect $\partial A_{\ell}$ at two points (since the distribution of $\left(\lambda_{1}, \lambda_{2}\right)$ has no atoms). We denote these points by $\left(d_{1}, d_{2}\right)$ and $\left(e_{1}, e_{2}\right)$ and without loss of generality take $d_{1}<e_{1}$. We now consider the region $\left\{x \leq e_{1}\right\} \cap\left\{y \leq d_{2}\right\}$. Reasoning as before, conditioning on 


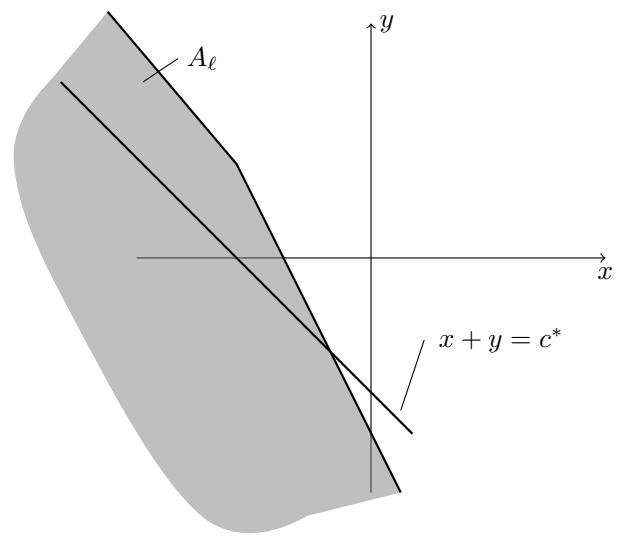

(a)

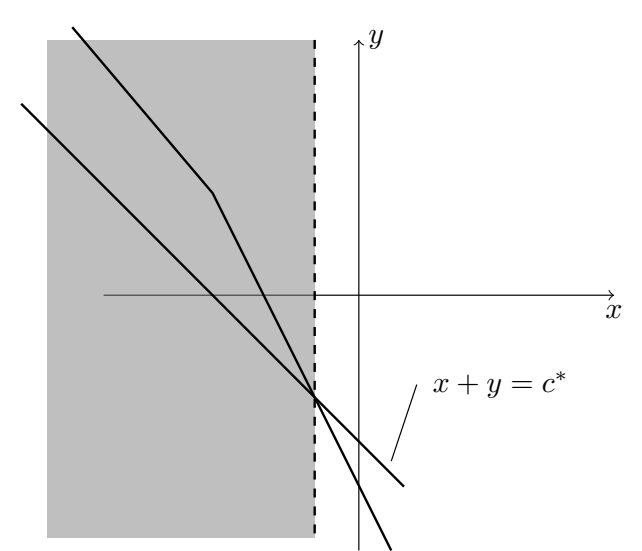

(b)

Figure 6: When $A_{\ell}$ takes the form shown in (a) (Case 2), we condition on the region shown in (b), which weakly increases the mean of $\lambda_{1}+\lambda_{2}$.

$\left(\lambda_{1}, \lambda_{2}\right)$ lying in this region weakly increases the probability that $\lambda_{1}+\lambda_{2}=c$ for $c \geq c^{*}$ and weakly decreases this probability for $c<c^{*}$ (see Figure 7). So in this case

$$
\mathbb{E}\left(\lambda_{1}+\lambda_{2} \mid\left(\lambda_{1}, \lambda_{2}\right) \in A_{\ell}\right) \leq \mathbb{E}\left(\lambda_{1}+\lambda_{2} \mid \lambda_{1} \leq e_{1}, \lambda_{2} \leq d_{2}\right) .
$$

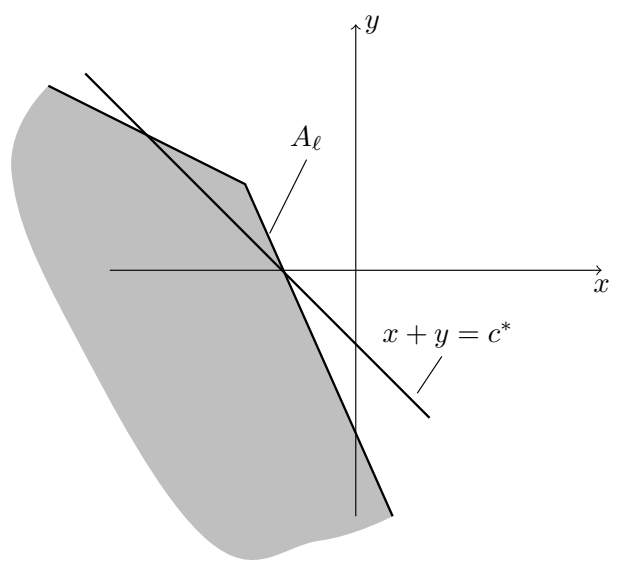

(a)

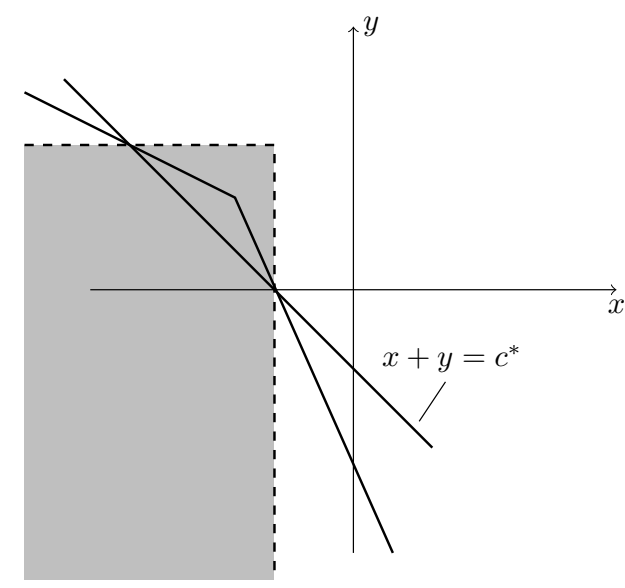

(b)

Figure 7: When $A_{\ell}$ takes the form shown in (a) (Case 3), we condition on the region shown in (b), which weakly increases the mean of $\lambda_{1}+\lambda_{2}$.

From (5.8), (5.9) and (5.10) we see that in order to complete the proof of the lemma, we need only verify (5.7) (or, equivalently, (5.6)) when $A_{\ell}$ is of the form $\left\{\lambda_{1} \leq c_{1}\right\}$, $\left\{\lambda_{2} \leq c_{2}\right\}$ or $\left\{\lambda_{1} \leq c_{1}, \lambda_{2} \leq c_{2}\right\}$. Furthermore, since $\lambda_{2} \geq 0 \geq \lambda_{1}$, we may assume $c_{1} \leq 0$ and $c_{2} \geq 0$.

Gromov's theorem ([14, Theorem 1.3]) states that if $h_{1}, h_{2}$ are integrable on $[a, b]$ such that $h_{2}>0$ and $h_{1}(x) / h_{2}(x)$ is non-increasing in $x$ then $\int_{a}^{c} h_{1}(x) d x / \int_{a}^{c} h_{2}(x) d x$ is non-increasing in $c$. Applying this to

$$
h_{1}(x):=\int_{-\infty}^{c_{2}} \frac{d}{d \ell} q_{\ell}(x, y) d y \text { and } h_{2}(x):=\int_{-\infty}^{c_{2}} q_{\ell}(x, y) d y
$$


we see that, provided $h_{1}(x) / h_{2}(x)$ is non-increasing, we have

$$
\frac{\int_{-\infty}^{c_{1}} \int_{-\infty}^{c_{2}} \frac{d}{d \ell} q_{\ell}(x, y) d y d x}{\int_{-\infty}^{c_{1}} \int_{-\infty}^{c_{2}} q_{\ell}(x, y) d y d x}
$$

is non-increasing in $c_{1}$. Then taking $c_{1} \rightarrow \infty$, we see that we need only verify (5.6) when $A_{\ell}$ is of the form $\left\{\lambda_{1} \leq c_{1}\right\}$ or $\left\{\lambda_{2} \leq c_{2}\right\}$.

It remains to show that $h_{1}(x) / h_{2}(x)$ is non-increasing in $x$, which is equivalent to $\mathbb{E}\left(\lambda_{1}+\lambda_{2} \mid \lambda_{1}=x, \lambda_{2} \leq c_{2}\right)$ being non-decreasing in $x$ for $x<0$. Using the joint density of $\left(\lambda_{1}, \lambda_{2}\right)$ given in Proposition 3.3

$$
\mathbb{E}\left(\lambda_{1}+\lambda_{2} \mid \lambda_{1}=x, \lambda_{2} \leq c_{2}\right)=\frac{\int_{0}^{c_{2}} y\left(y^{2}-x^{2}\right) \exp \left(-\frac{1}{2 \sigma^{2}}(y-m)^{2}\right) d y}{\int_{0}^{c_{2}} y(y-x) \exp \left(-\frac{1}{2 \sigma^{2}}(y-m)^{2}\right) d y}
$$

where $m=(1+\tau) \mu \ell-\tau x$. We next differentiate this expression with respect to $x$. To simplify the resulting expression, we let $Z$ be a random variable with density proportional to $\exp \left(-\frac{1}{2 \sigma^{2}}(y-m)^{2}\right) \mathbb{1}_{y \in\left[0, c_{2}\right]}$ (i.e. $Z$ is a truncated normal variable). Then using the Leibniz rule for differentiating integrals:

$$
\begin{aligned}
& \left(\frac{\int_{0}^{c_{2}} y(y-x) \exp \left(-\frac{1}{2 \sigma^{2}}(y-m)^{2}\right) d y}{\int_{0}^{c_{2}} \exp \left(-\frac{1}{2 \sigma^{2}}(y-m)^{2}\right) d y}\right)^{2}\left(\frac{d}{d x} \mathbb{E}\left(\lambda_{1}+\lambda_{2} \mid \lambda_{1}=x, \lambda_{2} \leq c_{2}\right)\right) \\
& =\left(\mathbb{E}\left(Z^{2}\right)-x \mathbb{E}(Z)\right)\left(-3 \tau \mathbb{E}\left(Z^{2}\right)-2 x \mathbb{E}(Z)+\tau x^{2}+\frac{\tau c_{2}\left(c_{2}^{2}-x^{2}\right) e^{-\frac{\left(c_{2}-m\right)^{2}}{2 \sigma^{2}}}}{\int_{0}^{c_{2}} \exp \left(-(y-m)^{2} /\left(2 \sigma^{2}\right)\right) d y}\right) \\
& -\left(\mathbb{E}\left(Z^{3}\right)-x^{2} \mathbb{E}(Z)\right)\left(-(2 \tau+1) \mathbb{E}(Z)+\tau x+\frac{\tau c_{2}\left(c_{2}-x\right) e^{-\frac{\left(c_{2}-m\right)^{2}}{2 \sigma^{2}}}}{\int_{0}^{c_{2}} \exp \left(-(y-m)^{2} /\left(2 \sigma^{2}\right)\right) d y}\right) .
\end{aligned}
$$

This expression can be rearranged to take the form

$$
\begin{aligned}
& \underbrace{(2 \tau+1) \mathbb{E}\left(Z^{3}\right) \mathbb{E}(Z)-3 \tau \mathbb{E}\left(Z^{2}\right)^{2}}_{A}+\underbrace{\tau \mathbb{E}\left(c_{2}^{2} Z^{2}\left(c_{2}-Z\right)\right) \frac{e^{-\frac{\left(c_{2}-m\right)^{2}}{2 \sigma^{2}}}}{\int_{0}^{c_{2}} e^{-\frac{(y-m)^{2}}{2 \sigma^{2}}} d y}}_{B} \\
& -x(\underbrace{(2-3 \tau) \mathbb{E}\left(Z^{2}\right) \mathbb{E}(Z)+\tau \mathbb{E}\left(Z^{3}\right)}_{C}+\underbrace{\tau \mathbb{E}\left(c_{2} Z\left(c_{2}^{2}-Z^{2}\right)\right) \frac{e^{-\frac{\left(c_{2}-m\right)^{2}}{2 \sigma^{2}}}}{\int_{0}^{c_{2}} e^{-\frac{(y-m)^{2}}{2 \sigma^{2}}} d y}}_{D}) \\
& +x^{2}(\underbrace{\tau \mathbb{E}\left(Z^{2}\right)+(1-2 \tau) \mathbb{E}(Z)^{2}}_{E}+\underbrace{\tau c_{2} \mathbb{E}\left(Z\left(c_{2}-Z\right)\right) \frac{e^{-\frac{\left(c_{2}-m\right)^{2}}{2 \sigma^{2}}}}{\int_{0}^{c_{2}} e^{-\frac{(y-m)^{2}}{2 \sigma^{2}}} d y}}_{F}) .
\end{aligned}
$$

Then, since $x<0$, we need only verify that each of the terms $A-F$ are non-negative. We show this by using two facts: first, that $0 \leq Z \leq c_{2}$ (which is true by definition) and second, that $0 \leq \tau \leq 1$ (which holds because $\tau=\left(\chi^{2}-1\right) /\left(3-\chi^{2}\right)$ and Assumption 2.15 implies that $1 \leq \chi^{2} \leq 2$ ).

These two facts immediately show that $B, D$ and $F$ are non-negative. Furthermore

$$
\begin{aligned}
& A \geq(2 \tau+1)\left(\mathbb{E}\left(Z^{3}\right) \mathbb{E}(Z)-\mathbb{E}\left(Z^{2}\right)^{2}\right) \\
& C=2(1-\tau) \mathbb{E}\left(Z^{2}\right) \mathbb{E}(Z)+\tau \operatorname{Cov}\left(Z^{2}, Z\right) \\
& E=(1-\tau) \mathbb{E}(Z)^{2}+\tau \operatorname{Var}(Z)
\end{aligned}
$$


(where the first inequality uses $\tau \leq 1$ ). Applying the Cauchy-Schwarz inequality to $Z^{2}=Z^{3 / 2} Z^{1 / 2}$ shows that $A$ is non-negative. Using the fact that $Z \geq 0$ (so that $Z^{2}$ is an increasing function of $Z$ ) implies that $C \geq 0$. Since $\tau \in[0,1]$ we see that $E \geq 0$.

Using Gromov's theorem in the same way as above, shows that in order to verify (5.7) for $A_{\ell}$ of the form $\left\{\lambda_{2} \leq c_{2}\right\}$ or $\left\{\lambda_{1} \leq c_{1}\right\}$ it is enough to show that $\mathbb{E}\left(\lambda_{1}+\lambda_{2} \mid \lambda_{2}=c_{2}\right)$ and $\mathbb{E}\left(\lambda_{1}+\lambda_{2} \mid \lambda_{1}=c_{1}\right)$ are non-decreasing in $c_{2}>0$ and $c_{1}<0$ respectively. This can be proven using a near identical calculation to that for $\frac{d}{d x} \mathbb{E}\left(\lambda_{1}+\lambda_{2} \mid \lambda_{1}=x, \lambda_{2} \leq c_{2}\right)$ (the only change is the region on which $Z$ is truncated, which means there will be no terms analogous to $B, D$ and $F$ above). This completes the proof of the lemma.

Remark 5.8. In Assumption 2.15, we impose the condition that $\chi \geq 1$. The only point in this paper at which we use this condition is in the proof of Lemma 5.7, in order to show that

$$
\mathbb{E}\left(\lambda_{1}+\lambda_{2} \mid \lambda_{1}=x, \lambda_{2} \leq c_{2}\right), \quad \mathbb{E}\left(\lambda_{1}+\lambda_{2} \mid \lambda_{1}=x\right), \quad \text { and } \quad \mathbb{E}\left(\lambda_{1}+\lambda_{2} \mid \lambda_{2}=x\right)
$$

are non-decreasing in $x$ (for all $c_{2} \geq 0$ ). Therefore, if an alternative method was found to verify this property (or to verify that (5.7) holds for $A_{\ell}$ of the form $\left\{\lambda_{1} \leq c_{1}\right\},\left\{\lambda_{2} \leq c_{2}\right\}$ and $\left\{\lambda_{1} \leq c_{1}, \lambda_{2} \leq c_{2}\right\}$ ) for fields with $\chi<1$, then our results (including Theorem 2.16) would also hold for such fields.

We expect that it should be possible to extend our results in this way. In the proof of Lemma 5.7 we use $\chi \geq 1$ (or equivalently, $\tau \geq 0$ ) to show that $A-F$ are non-negative. If we explicitly evaluate these terms using the higher order moments of a truncated normal distribution, then numerical calculations suggest that $A+B, C+D$ and $E+F$ are non-negative for all relevant values of $\tau$, (i.e. including negative values) which would be sufficient to prove this lemma in such cases. We do not attempt to prove this analytically, because the algebraic expressions involved in these calculation are quite long and we are primarily interested in the case of the BF field, for which $\tau=0$.

\subsection{Proof of Theorem 2.16}

We now use Lemmas 5.5 and 5.7 to complete the proof of Theorem 2.16, treating the RPW case and the general case simultaneously.

Proof of Theorem 2.16. We begin by fixing a realisation of $g$ and $\theta$. Let $A(\epsilon, R)$ denote the annulus on the plane centred at the origin with inner radius $\epsilon$ and outer radius $R$. We discretise this region by considering the points with polar coordinates

$$
\left(r_{i}^{(n)}, \omega_{j}^{(n)}\right):=\left(r_{i}, \omega_{j}\right):=\left(\epsilon+i 2^{-n}(R-\epsilon), \theta+j 2^{-n} 2 \pi\right)
$$

for $i, j=0,1, \ldots, 2^{n}$. We consider these points as a graph by placing an edge between $\left(r_{i_{1}}, \omega_{j_{1}}\right)$ and $\left(r_{i_{2}}, \omega_{j_{2}}\right)$ if and only if $\left|i_{1}-i_{2}\right|+\left|j_{1}-j_{2}\right|=1$. We define a site percolation model by declaring the vertex $\left(r_{i}, \omega_{j}\right)$ open if $\tilde{f}_{\ell}\left(r_{i}, \omega_{j}\right)-\ell<0$ (so an edge is open precisely when both of its vertices are open). Let $S_{\epsilon, R, n, \ell}$ denote the event that there is an open path between $(\epsilon, \theta)$ and $(\epsilon, \theta+\pi)$ in this percolation model.

Let $S_{\epsilon, R, \ell}$ denote the event that $\left\{\tilde{f}_{\ell}<\ell\right\} \cap A(\epsilon, R)$ contains a path joining $(\epsilon, \theta)$ to $(\epsilon, \theta+\pi)$. We claim that with probability one,

$$
\mathbb{1}_{S_{\epsilon, R, \ell}}=\lim _{n \rightarrow \infty} \mathbb{1}_{S_{\epsilon, R, n, \ell}} .
$$

Since $\tilde{f}_{\ell}$ has no critical points at level $\ell$ away from the origin (Lemma 4.3), the level set $\left\{\tilde{f}_{\ell}=\ell\right\} \cap A(\epsilon, R)$ consists of $C^{2+\eta}$ curves. So in particular, if there exists a path in $\left\{\tilde{f}_{\ell}<\ell\right\} \cap A(\epsilon, R)$ joining $(\epsilon, \theta)$ to $(\epsilon, \theta+\pi)$, then for $n$ sufficiently large we may assume this path lies on the graph with vertices $\left(r_{i}^{(n)}, \omega_{j}^{(n)}\right)$ as defined above. Hence 
$\mathbb{1}_{S_{\epsilon, R, \ell}} \leq \liminf _{n} \mathbb{1}_{S_{\epsilon, R, n, \ell}}$. If there is no path in $\left\{\tilde{f}_{\ell}<\ell\right\} \cap A(\epsilon, R)$ joining $(\epsilon, \theta)$ to $(\epsilon, \theta+\pi)$ then there are three possibilities: (1) $\tilde{f}_{\ell}-\ell$ is non-negative at $(\epsilon, \theta)$ or $(\epsilon, \theta+\pi)$; (2) there exists a path in $\left\{\tilde{f}_{\ell} \geq \ell\right\} \cap A(\epsilon, R)$ joining $\left(\epsilon, \omega_{i}\right)$ to $\left(\epsilon, \omega_{j}\right)$ for some $\omega_{i} \in(\theta, \theta+\pi)$ and $\omega_{j} \in(\theta-\pi, \theta)$, (here we note that by Lemma 4.3, $\left.\tilde{f}_{\ell}\right|_{\partial B(\epsilon)}$ has no local extrema at level $\ell$ and we assume that $n$ is sufficiently large to find such $\omega_{i}, \omega_{j}$ ); or (3) there exist two paths in $\left\{\tilde{f}_{\ell} \geq \ell\right\} \cap A(\epsilon, R)$ which join $\left(\epsilon, \omega_{i}\right)$ and $\left(\epsilon, \omega_{j}\right)$ respectively to $\partial B(R)$ for $\omega_{i}, \omega_{j}$ as before (See Figure 8 ). In this case, by Lemma 4.3 we may assume that the paths intersect $\partial B(R)$ at different points.

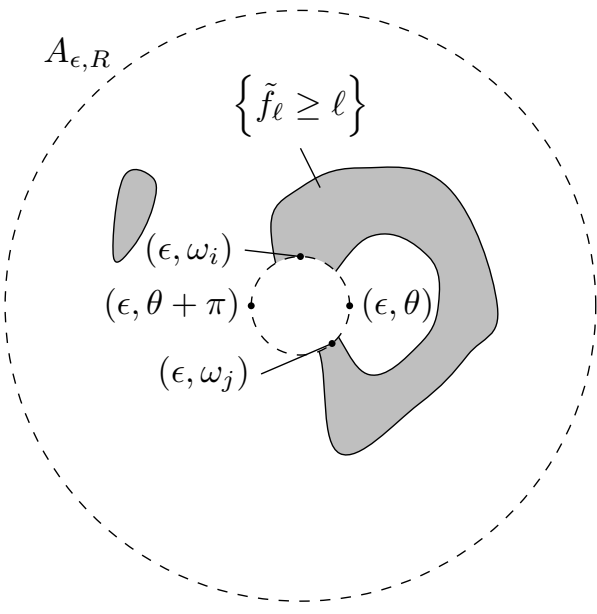

(a)

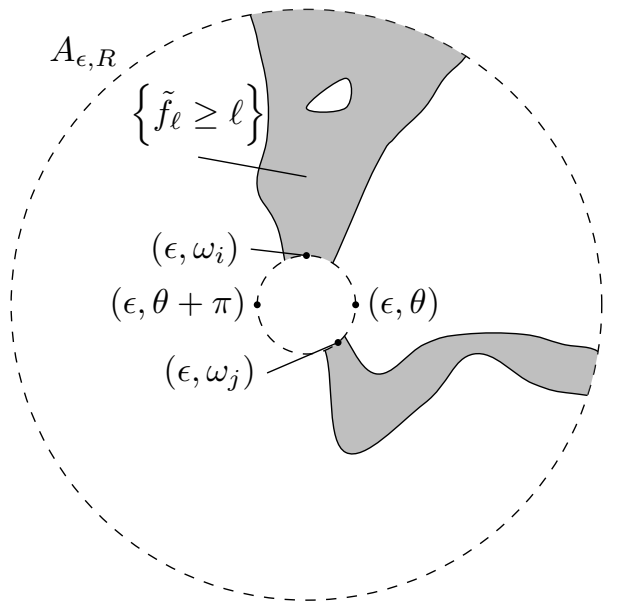

(b)

Figure 8: Two of the three ways in which $S_{\epsilon, R, \ell}$ can fail, corresponding to cases (2) and (3) above respectively.

In each of these cases, for all $n$ large enough we can construct corresponding paths on the discrete lattice as above which block a discrete path from joining $(\epsilon, \theta)$ to $(\epsilon, \theta+\pi)$ in $\left\{\tilde{f}_{\ell}<\ell\right\}$ and so $S_{\epsilon, R, n, \ell}$ cannot occur for sufficiently large $n$. Therefore $\mathbb{1}_{S_{\epsilon, R, \ell}} \geq \lim \sup _{n} \mathbb{1}_{S_{\epsilon, R, n, \ell}}$, completing the proof of the claim.

Since $S_{\epsilon, R, n, \ell}$ depends on only finitely many points of $\tilde{f}_{\ell}$ and is a decreasing event, by Lemma 5.5 for the RPW and Lemma 5.7 for general fields

$$
\mathbb{P}\left(S_{\epsilon, R, n, \ell_{1}} \mid g, \theta\right) \leq \mathbb{P}\left(S_{\epsilon, R, n, \ell_{2}} \mid g, \theta\right)
$$

for any $\ell_{1}<\ell_{2}$. Then by (5.11) and the bounded convergence theorem

$$
\mathbb{P}\left(S_{\epsilon, R, \ell_{1}} \mid g, \theta\right) \leq \mathbb{P}\left(S_{\epsilon, R, \ell_{2}} \mid g, \theta\right) .
$$

Now let $S_{R, \ell}$ be the event that $\tilde{f}_{\ell}$ has an $R$-lower connected saddle point at the origin. Conditional on $\theta$, if this event occurs then so must $S_{\epsilon, R, \ell}$ for $\epsilon$ sufficiently small. Conversely, if the saddle point at the origin is not $R$-lower connected, then it must be four-arm in $B(R)$ or $R$-upper connected. In both of these cases, $S_{\epsilon, R, \ell}$ cannot occur for $\epsilon$ sufficiently small. We conclude that $\mathbb{1}_{S_{R, \ell}}=\lim _{\epsilon \rightarrow 0} \mathbb{1}_{S_{\epsilon, R, \ell}}$ and by applying the bounded convergence theorem to (5.12) we see that

$$
\mathbb{P}\left(S_{R, \ell_{1}} \mid g, \theta\right) \leq \mathbb{P}\left(S_{R, \ell_{2}} \mid g, \theta\right) .
$$

Finally we let $S_{\ell}$ be the event that $\tilde{f}_{\ell}$ has a lower connected saddle point at the origin and note that trivially $S_{\ell}=\cup_{R} S_{R, \ell}$. Applying this to (5.13) shows that

$$
\mathbb{P}\left(S_{\ell_{1}} \mid g, \theta\right) \leq \mathbb{P}\left(S_{\ell_{2}} \mid g, \theta\right) .
$$


Integrating over realisations of $g$ and $\theta$ implies that $\mathbb{P}\left(S_{\ell_{1}}\right) \leq \mathbb{P}\left(S_{\ell_{2}}\right)$ and so by definition (see the proof of Theorem 2.11)

$$
\frac{p_{s^{-}}^{*}\left(\ell_{1}\right)}{p_{s}\left(\ell_{1}\right)} \leq \frac{p_{s^{-}}^{*}\left(\ell_{2}\right)}{p_{s}\left(\ell_{2}\right)}
$$

A near identical argument shows that $p_{s^{+}}^{*}(\ell) / p_{s}(\ell)$ is non-increasing in $\ell$.

\subsection{Remaining results}

We now prove the remaining results stated in Section 2.2, namely Corollaries 2.17 and 2.18 and Propositions 2.20-2.23.

Proof of Corollary 2.17. Since $p_{s^{-}}^{*} / p_{s}$ is monotone it has at most a countable number of discontinuities, all of which are jump discontinuities. By the continuity of $p_{s}$, the same is true of $p_{s^{-}}^{*}$. Since $c_{E S}$ is absolutely continuous (Theorem 2.6) it is differentiable almost everywhere (see [31, Theorem 7.18]) with derivative $p_{s^{-}}^{*}-p_{m^{+}}$. The density $p_{m^{+}}$is derived explicitly in [12] and is continuously differentiable. It also follows from monotonicity that $p_{s^{-}}^{*} / p_{s}$ is differentiable almost everywhere, and since $p_{s}$ is smooth (again, from [12]) the same is true of $p_{s^{-}}^{*}$, thus showing that $c_{E S}$ is twice differentiable almost everywhere. A similar proof applies to $c_{L S}$.

Proof of Corollary 2.18. Since the equivalence of (2)-(4) follows from Theorem 2.6, and (1) implies (2) by Theorem 2.11, it remains to show that (2) implies (1). Now suppose there exists a version of $p_{s^{-}}$which is continuous on $(a, b)$, denoted $\tilde{p}_{s^{-}}$. Then $\tilde{p}_{s^{-}} / p_{s}=$ $p_{s^{-}}^{*} / p_{s}$ almost everywhere, and since the former is continuous and the latter is monotone, this equality must hold pointwise on $(a, b)$, so $p_{s^{-}}^{*}$ is continuous on $(a, b)$. We note that $\tilde{p}_{s^{+}}:=p_{s}-\tilde{p}_{s^{-}}$defines a continuous version of $p_{s^{+}}$and arguing as above then shows that $p_{s^{+}}^{*}$ is continuous on $(a, b)$. Therefore the almost everywhere equality $p_{s^{-}}^{*}+p_{s^{+}}^{*}=p_{s}$ is in fact true for all points in $(a, b)$, and by (4.2) $\tilde{f}_{\ell}$ almost surely has no infinite four-arm saddle at the origin for all $\ell \in(a, b)$.

Proof of Proposition 2.23. We use the 'barrier method', that is, we show that the probability of having at least one component of $\{f \geq \ell\}$ contained in $B(r)$ is strictly positive for some fixed $r>0$. By linearity of expectation and stationarity of $f$, this shows that $\liminf _{R \rightarrow \infty} \mathbb{E}\left(N_{E S}(R, \ell)\right) / R^{2}>0$, so in particular $c_{E S}(\ell)>0$.

It is known that the RPW has the orthogonal expansion

$$
f(x)=\sum_{m \in \mathbb{Z}} a_{m} J_{|m|}(r) e^{i m \theta}
$$

where $(r, \theta)$ represents $x$ in polar coordinates, $J_{m}$ is the $m$-th Bessel function and $a_{m}=b_{m}+i c_{m}=\overline{a_{-m}}$ with $b_{0},\left(\sqrt{2} b_{m}\right)_{m \in \mathbb{N}}$ and $\left(\sqrt{2} c_{m}\right)_{m \in \mathbb{N}}$ independent standard (real) Gaussians and $c_{0}=0$. (This function is clearly Gaussian and can be shown to have the correct covariance structure using Graf's addition theorem for Bessel functions.) Let $r$ be the minimiser of $J_{0}$, so $r \approx 3.83$ and $J_{0}(r)<-0.4$. We note that by considering the power series for the Bessel functions, it can be shown that for $x \in[0,4],\left|J_{m}(x)\right| \leq e^{4}\left(2^{m} / m !\right)$. Finally we note that $J_{m}$ is bounded in absolute value by 1 for any $m$. Now consider the event that

$$
a_{0}>\min \{|\ell|, 1\} \quad \text { and }\left|a_{-1}\right|,\left|a_{-2}\right|,\left|a_{1}\right|,\left|a_{2}\right| \leq C_{1} \quad \text { and } \quad \forall|m|>2,\left|a_{m}\right| \leq C_{2}(m !) / 4^{m} .
$$

It is easily seen that this event has positive probability, and for appropriately chosen constants $C_{1}, C_{2}>0$, we see that on this event $f(0)>\ell$ and $f(x)<\ell$ for any $x$ such that $|x|=r$. Therefore $f$ has a component of $\{f \geq \ell\}$ contained in $B(r)$ with positive probability, completing the proof of the result. 
Proof of Proposition 2.20. By Corollary 2.18 we may assume that $p_{s^{-}}(\ell) / p_{s}(\ell)$ is nondecreasing. In [12] it is shown that for the RPW

$$
\begin{aligned}
p_{m^{+}}(x) & =\frac{1}{4 \sqrt{2} \pi^{3 / 2}}\left(\left(x^{2}-1\right) e^{-\frac{x^{2}}{2}}+e^{-\frac{3 x^{2}}{2}}\right) \mathbb{1}_{x \geq 0} \\
p_{s}(x) & =\frac{1}{4 \sqrt{2} \pi^{3 / 2}} e^{-\frac{3 x^{2}}{2}} .
\end{aligned}
$$

In particular, $p_{m^{+}}(x)=0$ for $x<0$, so by Theorem 2.6 for $\ell^{\prime}<\ell \leq 0$

$$
c_{E S}\left(\ell^{\prime}\right)-c_{E S}(\ell)=\int_{\ell^{\prime}}^{\ell}-p_{s^{-}}(x) d x .
$$

Taking $\ell^{\prime} \rightarrow-\infty$ shows that for $\ell<0$

$$
c_{E S}(\ell)=\int_{-\infty}^{\ell} p_{s^{-}}(x) d x
$$

By Proposition 2.23 this is positive for every $\ell<0$, so in particular there must exist arbitrarily negative $x$ such that $p_{s^{-}}(x)>0$. Since $p_{s^{-}}(\ell) / p_{s}(\ell)$ is non-decreasing, we conclude that $p_{s^{-}}$is strictly positive for all $\ell \in \mathbb{R}$. Since $p_{s^{-}}(x)=p_{s^{+}}(-x)$ we also see that $p_{s^{+}}(x)>0$ for all $x$ and since $p_{s^{-}}+p_{s^{+}}=p_{s}$ we see that $0<p_{s^{-}}(x) / p_{s}(x)<1$ for all $x \in \mathbb{R}$. Finally, we note that there must exist a sequence $\ell_{n}>0$ with $\ell_{n} \rightarrow 0$ such that $p_{s^{-}}\left(\ell_{n}\right) / p_{s}\left(\ell_{n}\right) \geq 1 / 2$ for all $n$. Indeed, if this were not true, by monotonicity, there would exist a neighbourhood of 0 on which $p_{s^{-}} / p_{s}<1 / 2$ and by symmetry $p_{s^{+}} / p_{s}<1 / 2$ on a possibly smaller neighbourhood, but then there would exist a set of positive measure on which $p_{s^{-}}+p_{s^{+}}<p_{s}$ giving a contradiction.

For $\ell^{\prime} \leq \ell$ and $\epsilon>0$

$$
\begin{aligned}
\frac{1}{\epsilon} \int_{\ell}^{\ell+\epsilon} \frac{p_{s^{-}}\left(\ell^{\prime}\right)}{p_{s}\left(\ell^{\prime}\right)} p_{s}(x)-p_{m^{+}}(x) d x & \leq \frac{1}{\epsilon} \int_{\ell}^{\ell+\epsilon} p_{s^{-}}(x)-p_{m^{+}}(x) d x \\
& \leq \frac{1}{\epsilon} \int_{\ell}^{\ell+\epsilon} \frac{p_{s^{-}}(\ell+\epsilon)}{p_{s}(\ell+\epsilon)} p_{s}(x)-p_{m^{+}}(x) d x
\end{aligned}
$$

By Theorem 2.6 and continuity of $p_{s}$ we therefore see that

$$
\frac{p_{s^{-}}\left(\ell^{\prime}\right)}{p_{s}\left(\ell^{\prime}\right)} p_{s}(\ell)-p_{m^{+}}(\ell) \leq D_{+} c_{E S}(\ell) \leq D^{+} c_{E S}(\ell) \leq \frac{p_{s^{-}}(\ell+\epsilon)}{p_{s}(\ell+\epsilon)} p_{s}(\ell)-p_{m^{+}}(\ell) .
$$

Since $p_{s^{-}} / p_{s}<1$, evaluating the final inequality using the explicit forms of $p_{s}$ and $p_{m^{+}}$ shows that $D^{+} c_{E S}(\ell)<0$ whenever $\ell \geq 1$. Since $p_{s^{-}}>0$ and $p_{m^{+}}(\ell)=0$ for $\ell \leq 0$, taking $\ell^{\prime}=\ell$ in the first inequality shows that $D_{+} c_{E S}(\ell)>0$ for $\ell \leq 0$. If $\ell>0$ then we may take $\ell^{\prime}=\ell_{n}$ as defined above for sufficiently large $n$. Then by evaluating the densities explicitly we see that $1 / 2 p_{s}(\ell)-p_{m^{+}}(\ell)>0$ for $\ell \in(0,0.876]$ thus completing the proof of the statements for $c_{E S}$.

Since $p_{m^{-}}(x)=0$ for $x>0$, we see from Theorem 2.6 that

$$
\frac{c_{L S}(\ell+\epsilon)-c_{L S}(\ell)}{\epsilon}=\frac{c_{E S}(\ell+\epsilon)-c_{E S}(\ell)}{\epsilon}-\frac{1}{\epsilon} \int_{\ell}^{\ell+\epsilon} p_{s^{+}}(x) d x
$$

for $\ell>0$. As $D^{+} c_{E S}(\ell)<0$ for $\ell \geq 1$ and $p_{s^{+}} \geq 0$, taking the limit superior here shows that $D^{+} c_{L S}(\ell)<0$ (for $\ell \geq 1$ ).

Proof of Propositions 2.21 and 2.22. By Theorem 2.10, both $c_{E S}$ and $c_{L S}$ are differentiable and so by Theorem 2.6

$$
\begin{aligned}
& c_{E S}^{\prime}(\ell)=p_{s^{-}}(\ell)-p_{m^{+}}(\ell) \leq p_{s}(\ell)-p_{m^{+}}(\ell) \\
& c_{L S}^{\prime}(\ell)=p_{m^{-}}(\ell)+p_{s^{-}}(\ell)-p_{m^{+}}(\ell)-p_{s^{+}}(\ell) \leq p_{m^{-}}(\ell)+p_{s}(\ell)-p_{m^{+}}(\ell) .
\end{aligned}
$$


The densities $p_{m^{-}}, p_{s}$ and $p_{m^{+}}$were derived for isotropic fields satisfying (a weaker version of) Assumption 2.1 in [12]. In the proof of [6, Corollary 1.19] it is shown that both right hand expressions in (5.14) are strictly negative whenever $\ell>\sqrt{2} / \chi$ (with $\chi$ defined prior to the statement of this proposition). We note that this is a sufficient condition for the derivatives to be negative, chosen for its simplicity. For many fields, the derivatives will be negative on a larger region and this can be found by using the densities specified in [12] with the appropriate value of $\chi$. Specifically, these densities are given in terms of $\chi$ and $\xi^{2}:=-k^{\prime}(0) / k^{\prime \prime}(0)$ by

$$
\begin{gathered}
p_{m^{+}}(x)=p_{m^{-}}(-x)=\frac{1}{\pi \xi^{2}}\left(\chi^{2}\left(x^{2}-1\right) \phi(x) \Phi\left(\frac{\chi x}{\sqrt{2-\chi^{2}}}\right)+\frac{\chi x \sqrt{2-\chi^{2}}}{2 \pi} e^{-\frac{x^{2}}{2-\chi^{2}}}\right. \\
\left.+\frac{\sqrt{2}}{\sqrt{\pi\left(3-\chi^{2}\right)}} e^{-\frac{3 x^{2}}{2\left(3-\chi^{2}\right)}} \Phi\left(\frac{\chi x}{\sqrt{\left(3-\chi^{2}\right)\left(2-\chi^{2}\right)}}\right)\right) \\
p_{s}(x)=\frac{1}{\pi \xi^{2}} \frac{\sqrt{2}}{\sqrt{\pi\left(3-\chi^{2}\right)}} e^{-\frac{3 x^{2}}{2\left(3-\chi^{2}\right)}}
\end{gathered}
$$

where $\phi$ and $\Phi$ denote the standard normal probability density and cumulative density respectively. For the Bargmann-Fock field, (for which $\chi=1$ ) substituting these densities into (5.14) shows that $c_{E S}^{\prime}(\ell), c_{L S}^{\prime}(\ell)<0$ for $\ell \geq 1.03$ improving on the general bound $\ell>\sqrt{2} / \chi=\sqrt{2}$.

Finally we note that $c_{E S}^{\prime}(0)=p_{s^{-}}(0)-p_{m^{+}}(0)$, and by the identities $p_{s^{-}}(x)=p_{s^{+}}(-x)$, $p_{s^{-}}+p_{s^{+}}=p_{s}$ almost everywhere and the fact these densities are all continuous, we see that $p_{s^{-}}(0)=p_{s}(0) / 2$. Evaluating the densities given in [12] at zero shows that $p_{s}(0) / 2>p_{m^{+}}(0)$ so we conclude that $c_{E S}^{\prime}(0)>0$. Since $c_{E S}$ is continuously differentiable, we can extend this to a neighbourhood of the origin.

By Theorem 2.16, $p_{s^{-}}(\ell) / p_{s}(\ell)$ is non-decreasing and so for $\ell>0$

$$
\frac{p_{s^{-}}(\ell)}{p_{s}(\ell)} \geq \frac{p_{s-}(0)}{p_{s}(0)}=\frac{1}{2}
$$

Therefore $c_{E S}^{\prime}(\ell) \geq p_{s}(\ell) / 2-p_{m^{+}}(\ell)$ for $\ell \geq 0$. Evaluating the densities above then gives an explicit constant $C$ such that this expression is strictly positive for $\ell \leq C$. In the case of the Bargmann-Fock field, $C=0.64$.

\section{A Non-degeneracy}

We verify some claims about the non-degeneracy of Gaussian fields:

Lemma A.1. Let $f$ be a $C^{2}$, stationary, planar Gaussian field. Then the spectral measure $\mu$ being supported on the union of two lines through the origin is equivalent to the Gaussian vector $\nabla^{2} f(0)$ being degenerate.

Proof. By [1, Chapter 5], for any $s, t \in \mathbb{R}^{2}$ and $\alpha, \beta, \gamma, \delta \in \mathbb{N} \cup\{0\}$ (such that the following derivatives are defined)

$$
\mathbb{E}\left(\frac{\partial^{\alpha+\beta}}{\partial t_{1}^{\alpha} \partial t_{2}^{\beta}} f(t) \overline{\frac{\partial^{\gamma+\delta}}{\partial s_{1}^{\gamma} \partial s_{2}^{\delta}} f(s)}\right)=\int_{\mathbb{R}^{2}}\left(-i x_{1}\right)^{\alpha}\left(-i x_{2}\right)^{\beta} e^{-i t \cdot x} \overline{\left(-i x_{1}\right)^{\gamma}\left(-i x_{2}\right)^{\delta} e^{-i s \cdot x}} d \mu(x)
$$

where $\mu$ is the spectral measure of $f$. Then for $a \in \mathbb{R}^{3}$,

$$
\mathbb{E}\left(\left(a \cdot \nabla^{2} f(0)\right)^{2}\right)=\int_{\mathbb{R}^{2}}\left|a_{1} x_{1}^{2}+a_{2} x_{2}^{2}+a_{3} x_{1} x_{2}\right|^{2} d \mu(x) .
$$


If $\nabla^{2} f(0)$ is degenerate, then we may choose $a \neq 0$ such that this expression is zero, and hence the integrand is identically zero on the support of $\mu$. Hence the support of $\mu$ is contained in the zero set of this binary quadratic form which is contained in the union of two lines through the origin.

Conversely if the support of $\mu$ is contained in the union of two lines through the origin, then we may choose $a \neq 0$ such that the zero set of $a_{1} x_{1}^{2}+a_{2} x_{2}^{2}+a_{3} x_{1} x_{2}$ is equal to this union. Hence the integral above will be zero and $\nabla^{2} f(0)$ will be degenerate.

Lemma A.2. Let $f: \mathbb{R}^{2} \rightarrow \mathbb{R}$ be a Gaussian field which is stationary and centred with $\operatorname{Var}(f(0))=1$ and covariance function $K \in C^{4+\eta^{\prime}}$. If the support of the spectral measure $\mu$ contains a centred ellipse (or circle), then Assumptions 2.1 and 2.7 hold. Moreover, if the support of $\mu$ contains an open set then, for any distinct $t_{1}, \ldots, t_{n} \subset \mathbb{R}^{2}$, the vector

$$
\left(f\left(t_{1}\right), \ldots, f\left(t_{n}\right), \nabla f\left(t_{1}\right), \ldots, \nabla f\left(t_{n}\right), \nabla^{2} f\left(t_{1}\right), \ldots, \nabla^{2} f\left(t_{n}\right)\right)
$$

is non-degenerate (so in particular, Assumptions 2.1 and 2.7 hold).

We note that these results could be proven under much weaker conditions on the support of the spectral measure using the arguments we give below. We do not attempt to formulate the most general conditions.

Proof. First consider the case that the support of $\mu$ contains an ellipse/circle. Let $a \in \mathbb{R}^{9}$ and

$$
w:=\left(f(t), \nabla f(t), f(0), \nabla f(0), \nabla^{2} f(0)\right) .
$$

By the same arguments as in the proof of Lemma A.1

$$
\mathbb{E}\left((a \cdot w)^{2}\right)=\int_{\mathbb{R}^{2}}\left|a \cdot\left(-e^{-i t \cdot x}, i x_{1} e^{-i t \cdot x}, i x_{2} e^{-i t \cdot x},-1, i x_{1}, i x_{2}, x_{1}^{2}, x_{1} x_{2}, x_{2}^{2}\right)\right|^{2} d \mu(x) .
$$

If Assumption 2.7 does not hold, then there exists a choice of $a$ such that this expectation is zero and one of the first three elements of $a$ is non-zero. Hence the integrand above must be identically zero on the support of $\mu$. By considering the real and imaginary parts explicitly, the zero set of this integrand cannot contain an ellipse/circle centred at the origin and so neither can the support of $\mu$. By a near-identical argument, and Lemma A.1, $f$ also satisfies the non-degeneracy conditions of Assumption 2.1. (The other conditions are satisfied by the premise of this lemma.)

By a completely analogous argument we see that if

$$
\left(f\left(t_{1}\right), \ldots, f\left(t_{n}\right), \nabla f\left(t_{1}\right), \ldots, \nabla f\left(t_{n}\right), \nabla^{2} f\left(t_{1}\right), \ldots, \nabla^{2} f\left(t_{n}\right)\right)
$$

is degenerate then some non-trivial linear combination of

$$
\begin{aligned}
& e^{-i t_{1} \cdot x}, \ldots, e^{-i t_{n} \cdot x} \\
& i x_{1} e^{-i t_{1} \cdot x}, \ldots, i x_{1} e^{-i t_{n} \cdot x}, i x_{2} e^{-i t_{1} \cdot x}, \ldots, i x_{2} e^{-i t_{n} \cdot x} \\
& x_{1}^{2} e^{-i t_{1} \cdot x}, \ldots, x_{1}^{2} e^{-i t_{n} \cdot x}, x_{2}^{2} e^{-i t_{1} \cdot x}, \ldots, x_{2}^{2} e^{-i t_{n} \cdot x}, x_{1} x_{2} e^{-i t_{1} \cdot x}, \ldots, x_{1} x_{2} e^{-i t_{n} \cdot x}
\end{aligned}
$$

is identically zero on the support of $\mu$. Since the $t_{i}$ are distinct, we see that the support of $\mu$ cannot contain an open set.

Lemma A.3. Let $f$ be a Gaussian field satisfying Assumption 2.1. Then the density of saddle points $p_{s}$ defined in Proposition 2.5 is non-zero for all $\ell \in \mathbb{R}$.

Proof. By the Kac-Rice theorem (Corollary 11.2.2 of [1]), and the independence of $\nabla f(0)$ and $\left(f(0), \nabla^{2} f(0)\right)$,

$$
p_{s}(\ell)=\mathbb{E}\left[\left|\operatorname{det} \nabla^{2} f(0)\right| \mathbb{1}_{\operatorname{det} \nabla^{2} f(0)<0} \mid f(0)=\ell\right] p_{f(0)}(\ell) .
$$


We note that by Gaussian regression

$$
\operatorname{Cov}\left(\nabla^{2} f(0) \mid f(0)=\ell\right)=\operatorname{Cov}\left(\nabla^{2} f(0)\right)-\operatorname{Cov}\left(\nabla^{2} f(0), f(0)\right) \operatorname{Cov}\left(\nabla^{2} f(0), f(0)\right)^{t}
$$

where $\operatorname{Cov}\left(\nabla^{2} f(0)\right)$ is a three by three matrix and $\operatorname{Cov}\left(\nabla^{2} f(0), f(0)\right)$ is a three-dimensional row vector. Since we assume that $\nabla^{2} f(0)$ is non-degenerate, the conditional covariance matrix above is the difference between a rank three and rank one matrix, so must have rank at least two. Therefore $\left(\nabla^{2} f(0) \mid f(0)=\ell\right)$ must be supported on either a two or three dimensional (affine) subspace of $\mathbb{R}^{3}$. This implies that the support of

$$
\left(\operatorname{det} \nabla^{2} f(0) \mid f(0)=\ell\right)
$$

is $\mathbb{R}$, and hence by (A.1) $p_{s}(\ell)>0$ for all $\ell \in \mathbb{R}$.

\section{References}

[1] Robert J. Adler and Jonathan E. Taylor, Random fields and geometry, Springer Monographs in Mathematics, Springer, New York, 2007. MR-2319516

[2] Robert J. Adler, Jonathan E. Taylor, and Keith J. Worsley, Applications of random fields and geometry: foundations and case studies, In preparation, available at https://web.stanford. edu/class/stats317/hrf.pdf.

[3] Jean-Marc Azaïs and Mario Wschebor, Level sets and extrema of random processes and fields, John Wiley \& Sons, Inc., Hoboken, NJ, 2009. MR-2478201

[4] Vincent Beffara and Damien Gayet, Percolation of random nodal lines, Publ. Math. Inst. Hautes Études Sci. 126 (2017), 131-176. MR-3735866

[5] Dmitry Beliaev, Michael McAuley, and Stephen Muirhead, Fluctuations of the number of excursion sets of planar Gaussian fields, arXiv preprint arXiv:1908.10708 (2019).

[6] Dmitry Beliaev, Michael McAuley, and Stephen Muirhead, On the number of excursion sets of planar Gaussian fields, Probab. Theory Related Fields (2020).

[7] Dmitry Beliaev, Stephen Muirhead, and Igor Wigman, Russo-Seymour-Welsh estimates for the Kostlan ensemble of random polynomials, arXiv preprint arXiv:1709.08961 (2017).

[8] Dmitry Beliaev and Igor Wigman, Volume distribution of nodal domains of random bandlimited functions, Probab. Theory Related Fields 172 (2018), no. 1-2, 453-492. MR-3851836

[9] Carol Bezuidenhout, Geoffrey Grimmett, and Armin Löffler, Percolation and minimal spanning trees, J. Statist. Phys. 92 (1998), no. 1-2, 1-34. MR-1645627

[10] S. Bochner, Monotone Funktionen, Stieltjessche Integrale und harmonische Analyse, Math. Ann. 108 (1933), no. 1, 378-410. MR-1512856

[11] Eugene Bogomolny and Charles Schmit, Percolation model for nodal domains of chaotic wave functions, Physical Review Letters 88 (2002), no. 11, 114102.

[12] Dan Cheng and Armin Schwartzman, Expected number and height distribution of critical points of smooth isotropic Gaussian random fields, Bernoulli 24 (2018), no. 4B, 3422-3446. MR-3788177

[13] Mark R. Dennis, Nodal densities of planar Gaussian random waves, Eur. Phys. J. Spec. Top. 145 (2007), no. 1, 191-210.

[14] Ricardo Estrada and Miroslav Pavlović, L'hôpital's monotone rule, Gromov's theorem, and operations that preserve the monotonicity of quotients, Publ. Inst. Math. (Beograd) (N.S.) 101(115) (2017), 11-24. MR-3700399

[15] Christophe Garban and Jeffrey E. Steif, Noise sensitivity of Boolean functions and percolation, Institute of Mathematical Statistics Textbooks, vol. 5, Cambridge University Press, New York, 2015. MR-3468568

[16] Geoffrey Grimmett, Percolation, second ed., Grundlehren der Mathematischen Wissenschaften [Fundamental Principles of Mathematical Sciences], vol. 321, Springer-Verlag, Berlin, 1999. MR-1707339 
Smoothness/monotonicity of the excursion set density of Gaussian fields

[17] Martin Hairer, An introduction to stochastic PDEs, arXiv preprint arXiv:0907.4178 (2009). MR-2832168

[18] Maxime Ingremeau, Lower bounds for the number of nodal domains for sums of two distorted plane waves in non-positive curvature, Asian Journal of Mathematics (To appear).

[19] Sudhir Ranjan Jain and Rhine Samajdar, Nodal portraits of quantum billiards: Domains, lines, and statistics, Rev. Mod. Phys. 89 (2017), no. 4, 045005. MR-3746353

[20] Mark Kac and David Slepian, Large excursions of Gaussian processes, Ann. Math. Statist. 30 (1959), 1215-1228. MR-0115222

[21] Olav Kallenberg, Foundations of modern probability, second ed., Probability and its Applications (New York), Springer-Verlag, New York, 2002. MR-1876169

[22] Pär Kurlberg and Igor Wigman, Variation of the Nazarov-Sodin constant for random plane waves and arithmetic random waves, Adv. Math. 330 (2018), 516-552. MR-3787552

[23] James Clerk Maxwell, On hills and dales, The London, Edinburgh, and Dublin Philosophical Magazine and Journal of Science 40 (1870), no. 269, 421-427.

[24] John Milnor, Morse theory, Based on lecture notes by M. Spivak and R. Wells. Annals of Mathematics Studies, No. 51, Princeton University Press, Princeton, N.J., 1963. MR-0163331

[25] Stephen Muirhead and Hugo Vanneuville, The sharp phase transition for level set percolation of smooth planar Gaussian fields, Ann. Inst. Henri Poincaré Probab. Stat. 56 (2020), no. 2, 1358-1390. MR-4076787

[26] Fedor Nazarov and Mikhail Sodin, Asymptotic laws for the spatial distribution and the number of connected components of zero sets of Gaussian random functions, Zh. Mat. Fiz. Anal. Geom. 12 (2016), no. 3, 205-278. MR-3522141

[27] Andriy Ya. Olenko, Upper bound on $\sqrt{x} J_{v}(x)$ and its applications, Integral Transforms Spec. Funct. 17 (2006), no. 6, 455-467. MR-2238583

[28] Pratyush Pranav, Robert J. Adler, Thomas Buchert, Herbert Edelsbrunner, Bernard J. T. Jones, Armin Schwartzman, Hubert Wagner, and Rien van de Weygaert, Unexpected topology of the temperature fluctuations in the cosmic microwave background, A\&A 627 (2019), A163.

[29] Alejandro Rivera and Hugo Vanneuville, Quasi-independence for nodal lines, Ann. Inst. Henri Poincaré Probab. Stat. 55 (2019), no. 3, 1679-1711. MR-4010948

[30] Alejandro Rivera and Hugo Vanneuville, The critical threshold for Bargmann-Fock percolation, Annales Henri Lebesgue 3 (2020), 169-215 (en). MR-4060853

[31] Walter Rudin, Real and complex analysis, third ed., McGraw-Hill Book Co., New York, 1987. MR-0924157

[32] Marco Scarsini, Multivariate stochastic dominance with fixed dependence structure, Oper. Res. Lett. 7 (1988), no. 5, 237-240. MR-0962593

[33] Igor Wigman, On the nodal lines of random and deterministic Laplace eigenfunctions, Spectral geometry, Proc. Sympos. Pure Math., vol. 84, Amer. Math. Soc., Providence, RI, 2012, pp. 285297. MR-2985322

[34] Keith J. Worsley, Sean Marrett, Peter Neelin, Alain C. Vandal, Karl J. Friston, and Alan C. Evans, A unified statistical approach for determining significant signals in images of cerebral activation, Hum. Brain Mapp. 4 (1996), no. 1, 58-73.

Acknowledgments. The authors thank an anonymous referee for their careful reading of the manuscript, for making us aware of [23] and, in particular, for pointing out an error in Lemma 4.4. The authors also thank Igor Wigman and Ben Hambly for helpful comments on a slightly different version of this work. The first author was supported by the Engineering \& Physical Sciences Research Council (EPSRC) Fellowship EP/M002896/1. 


\section{Electronic Journal of Probability Electronic Communications in Probability}

\section{Advantages of publishing in EJP-ECP}

- Very high standards

- Free for authors, free for readers

- Quick publication (no backlog)

- Secure publication $\left(\mathrm{LOCKSS}^{1}\right)$

- Easy interface (EJMS²)

\section{Economical model of EJP-ECP}

- Non profit, sponsored by $\mathrm{IMS}^{3}, \mathrm{BS}^{4}$, ProjectEuclid ${ }^{5}$

- Purely electronic

\section{Help keep the journal free and vigorous}

- Donate to the IMS open access fund ${ }^{6}$ (click here to donate!)

- Submit your best articles to EJP-ECP

- Choose EJP-ECP over for-profit journals

\footnotetext{
${ }^{1}$ LOCKSS: Lots of Copies Keep Stuff Safe http://www. lockss.org/

${ }^{2}$ EJMS: Electronic Journal Management System http://www.vtex.lt/en/ejms.html

${ }^{3}$ IMS: Institute of Mathematical Statistics http://www.imstat.org/

${ }^{4}$ BS: Bernoulli Society http://www. bernoulli-society.org/

${ }^{5}$ Project Euclid: https://projecteuclid.org/

${ }^{6}$ IMS Open Access Fund: http://www.imstat.org/publications/open.htm
} 